Article

\title{
Synthesis, Antifungal Activity, 3D-QSAR, and Molecular Docking Study of Novel Menthol-Derived 1,2,4-Triazole-thioether Compounds
}

\author{
Mei Huang ${ }^{1,2}$, Wen-Gui Duan ${ }^{1, *}$, Gui-Shan Lin ${ }^{1, *}$ and Bao-Yu Li ${ }^{1}$ \\ 1 School of Chemistry and Chemical Engineering, Guangxi University, Nanning 530004, China; \\ 1514404003@st.gxu.edu.cn (M.H.); 2114402005@st.gxu.edu.cn (B.-Y.L.) \\ 2 Guangxi Research Institute of Chemical Industry Co., Ltd., Nanning 530001, China \\ * Correspondence: wgduan@gxu.edu.cn (W.-G.D.); gslin@gxu.edu.cn (G.-S.L.); \\ Tel.: +86-771-323-3718 (W.-G.D.); Fax: +86-771-323-3718 (W.-G.D.)
}

Citation: Huang, M.; Duan, W.-G.; Lin, G.-S.; Li, B.-Y. Synthesis,

Antifungal Activity, 3D-QSAR, and Molecular Docking Study of Novel Menthol-Derived

1,2,4-Triazole-thioether Compounds. Molecules 2021, 26, 6948. https://

doi.org/10.3390/molecules26226948

Academic Editor: Alla P. Toropova

Received: 24 October 2021

Accepted: 16 November 2021

Published: 17 November 2021

Publisher's Note: MDPI stays neutral with regard to jurisdictional claims in published maps and institutional affiliations.

Copyright: (C) 2021 by the authors. Licensee MDPI, Basel, Switzerland. This article is an open access article distributed under the terms and conditions of the Creative Commons Attribution (CC BY) license (https:/ / creativecommons.org/licenses/by/ $4.0 /)$.

\begin{abstract}
A series of novel menthol derivatives containing 1,2,4-triazole-thioether moiety were designed, synthesized, characterized structurally, and evaluated biologically to explore more potent natural product-based antifungal agents. The bioassay results revealed that at $50 \mu \mathrm{g} / \mathrm{mL}$, some of the target compounds exhibited good inhibitory activity against the tested fungi, especially against Physalospora piricola. Compounds $5 \mathbf{b}\left(\mathrm{R}=o-\mathrm{CH}_{3} \mathrm{Ph}\right), 5 \mathbf{i}(\mathrm{R}=o-\mathrm{Cl} \mathrm{Ph}), 5 \mathbf{v}\left(\mathrm{R}=m, p-\mathrm{OCH}_{3} \mathrm{Ph}\right)$ and 5x ( $\mathrm{R}=\alpha$-furyl $)$ had inhibition rates of $93.3 \%, 79.4 \%$, and $79.4 \%$, respectively, against $P$. piricola, much better than that of the positive control chlorothalonil. Compounds $5 \mathbf{v}\left(\mathrm{R}=m, p-\mathrm{OCH}_{3} \mathrm{Ph}\right)$ and $5 \mathrm{~g}(\mathrm{R}=o-\mathrm{Cl} \mathrm{Ph})$ held inhibition rates of $82.4 \%$ and $86.5 \%$ against Cercospora arachidicola and Gibberella zeae, respectively, much better than that of the commercial fungicide chlorothalonil. Compound $\mathbf{5 b}$ $\left(\mathrm{R}=o-\mathrm{CH}_{3} \mathrm{Ph}\right.$ ) displayed antifungal activity of $90.5 \%$ and $83.8 \%$, respectively, against Colleterichum orbicalare and Fusarium oxysporum $\mathrm{f}$. sp. cucumerinum. Compounds $5 \mathrm{~m}(\mathrm{R}=o-\mathrm{I} \mathrm{Ph})$ had inhibition rates of $88.6 \%, 80.0 \%$, and $88.0 \%$, respectively, against F. oxysporum f. sp. cucumerinu, Bipolaris maydis and $\mathrm{C}$. orbiculare. Furthermore, compound $5 \mathbf{b}\left(\mathrm{R}=o-\mathrm{CH}_{3} \mathrm{Ph}\right)$ showed the best and broad-spectrum antifungal activity against all the tested fungi. To design more effective antifungal compounds against P. piricola, 3D-QSAR analysis was performed using the CoMFA method, and a reasonable 3D-QSAR model $\left(r^{2}=0.991, q^{2}=0.514\right)$ was established. The simulative binding pattern of the target compounds with cytochrome P450 14 $\alpha$-sterol demethylase (CYP51) was investigated by molecular docking.
\end{abstract}

Keywords: menthol; 1,2,4-triazole-thioether; antifungal activity; 3D-QSAR; molecular docking

\section{Introduction}

Fungicides have been widely used for controlling the disease caused by plant pathogens. However, the developed resistance of plant-pathogenic fungi to currently commercial fungicides has shown great threat to the production and quality of plants, which lead to the exploration of novel antifungal agents.

Peppermint (Mentha Canadensis Linnaeus) is an important herb spice plant mainly planted in Jiangsu and Anhui regions of China, as well as a traditional Chinese medicine. Menthol, $(1 R, 2 S, 5 R)$-2- isopropyl-5-methylcyclohexan-1-ol, is the dominant constituent of the essential oil which is obtained by steam distillation of the fresh leaves of peppermint [1]. The content of menthol in peppermint essential oil can be as high as 30\% to 55\% [2]. Because menthol is a monocyclic monoterpene alcohol with three chiral carbons, it can be employed to synthesize bioactive compounds except its use in flavors and fragrances. Menthol and its derivatives have a wide range of applications in medicine and agrochemical, such as anti-inflammatory [3], antimicrobial [4,5], antifungal [6], insecticidal [7], anticancer [8,9], analgesic [10,11], anesthetic [12] and penetration-enhancing activities [13]. Thus, menthol deserves further study for agrochemical or pharmaceutical use based on its bioactive property and chemical reactivity. 
On the other hand, 1,2,4-triazole, as the representative of five-membered unsaturated heterocycles, showed diverse biological activities, such as antimicrobial [14,15], antifungal [16-18], anticancer [19,20], insecticidal [21,22], herbicidal [23,24] and anti-inflammatory activities [25]. In particular, several compounds such as cytochrome P450 14 $\alpha$-sterol demethylase (CYP51) inhibitors in the FRAC classification of fungicides (http:/ / www.frac.info/ accessed on 7 November 2021) were found to contain 1,2,4-triazole moiety. In addition, thioether derivatives have considerable applications in agriculture and medicine because of their various bioactive activities such as insecticidal [26], antifungal, antibacterial [27] and anticancer [28] activities.

In continuation of our interest in the bioactive properties of natural product-based compounds [29-37], a series of novel menthol-derived 1,2,4-triazole-thioether compounds were synthesized by integrating bioactive 1,2,4-triazole and thioether moieties into the molecular skeleton of menthol. Structural characterization, antifungal evaluation, threedimensional quantitative structure-activity relationship (3D-QSAR) and molecular docking of the title compounds were also carried out.

\section{Results and Discussion}

\subsection{Synthesis and Characterization}

As illustrated in Scheme 1, (-)-menthyl-2-chloroacetate (2) was prepared by the $O$ acylation reaction of (-)-menthol (1) with chloroacetyl chloride. Meanwhile, a series of substituted aryl hydrazides were converted to the corresponding 5-substituted 1,2,4-triazole-3thiones (4). Lastly, a series of menthol-derived 1,2,4-triazole-thioether compounds (5a-z) were synthesized by the nucleophilic substitution of compounds 4 with 2 under alkaline condition.<smiles>[R]C(=O)NNN</smiles>

\footnotetext{
5a: R=Ph; 5b: R=o- $\mathrm{CH}_{3} \mathrm{Ph}$; $5 \mathrm{c}: \mathrm{R}=p-\mathrm{CH}_{3} \mathrm{Ph}$; $5 \mathrm{~d}: \mathrm{R}=0-\mathrm{OCH}_{3} \mathrm{Ph}$; $5 \mathrm{e}: \mathrm{R}=p-\mathrm{OCH}_{3} \mathrm{Ph} ; 5 \mathrm{f}: \mathrm{R}=0-\mathrm{Fh}$; 5g: R=m-F Ph; 5h: R=p-F Ph; 5i: R=o-Cl Ph; 5j: R=m-Cl Ph; 5k: R=p-Cl Ph; 5l: R=p-Br Ph; 5m: R=o-I Ph; 5n: R=p-I Ph; 5o: R=o-CF 3 Ph; 5p: R=m-CF 3 Ph; 5q: R=o-OH Ph; 5r: R=p-OH Ph; 5s: R=o-NH 2 Ph; 5t: R=p-NH $\mathrm{Nh}_{2} \mathrm{Pu}: \mathrm{R}=p-\mathrm{C}\left(\mathrm{CH}_{3}\right)_{3} \mathrm{Ph} ; 5 \mathrm{v}$ : R=m, $p-\mathrm{OCH}_{3} \mathrm{Ph} ; 5 \mathrm{w}: \mathrm{R}=m, m-\mathrm{OCH}_{3} \mathrm{Ph}$; 5x: $\mathrm{R}=\alpha$-furyl; $5 \mathrm{y}$ : $\mathrm{R}=\alpha$-thienyl; $5 \mathrm{z}$ : $\mathrm{R}=\beta$-pyridyl
}

Scheme 1. Synthesis of menthol-derived 1,2,4-triazole-thioether compounds 5a-5z. Reagents and conditions: (a) Chloroacetyl chloride, TEA, DMAP, $0{ }^{\circ} \mathrm{C}$; (b) Methyl isothiocyanate, $\mathrm{EtOH}, 80{ }^{\circ} \mathrm{C}$, $3 \mathrm{~h}$; $\mathrm{NaOH} 10 \%, 100{ }^{\circ} \mathrm{C}, 4 \mathrm{~h}$; (c) Sodium acetate trihydrate, $\mathrm{H}_{2} \mathrm{O}, \mathrm{EtOH}, 80{ }^{\circ} \mathrm{C}, 6 \mathrm{~h}$.

The structures of all the target compounds and the key intermediate 2 were characterized by FT-IR, ${ }^{1} \mathrm{H}$ NMR, ${ }^{13} \mathrm{C}$ NMR, electrospray mass spectrometry (ESI-MS) and elemental analysis, and the related spectra could be found in the Supplementary Materials. In the FTIR spectra of the target compounds, the characteristic absorption bands at about $1731 \mathrm{~cm}^{-1}$ were assigned to the stretching vibrations of $\mathrm{C}=\mathrm{O}$. The characteristic absorption bands at about $1457-1486 \mathrm{~cm}^{-1}$ were assigned to $\mathrm{C}=\mathrm{N}$ in the 1,2,4-triazole moiety. Additionally, the characteristic absorption bands in the region of $686-714 \mathrm{~cm}^{-1}$ was attributed to the vibrations of C-S-C. In the ${ }^{1} \mathrm{H}-\mathrm{NMR}$ spectra, the protons of benzene ring showed signals at 
6.57-7.95 ppm. The characteristic signals at 3.38-3.86 ppm were assigned to the methyl protons linked to the 1,2,4-triazole heterocycle. The methylene protons bonded to the $S$ atom displayed the signals at about $3.40 \mathrm{ppm}$. The characteristic signals at about $4.71 \mathrm{ppm}$ were assigned to the protons on the saturated carbon bonded to the $\mathrm{O}$ atom. The protons of the menthol moiety displayed signals in the range of $0.71-2.02 \mathrm{ppm}$. The ${ }^{13} \mathrm{C}$ NMR spectra of the target compounds showed peaks for the carbon atom of $\mathrm{C}=\mathrm{O}$ at about $168.00 \mathrm{ppm}$ and carbon atoms of the benzene ring at $96.62-163.92 \mathrm{ppm}$. For the 1,2,4-triazole moiety, the signals at $150.36-157.37 \mathrm{ppm}$ and $144.06-151.45 \mathrm{ppm}$ were assigned to the unsaturated carbons, and at $31.41-33.40 \mathrm{ppm}$ to the methyl. The methylene carbon bonded to the $\mathrm{S}$ atom showed signals at $35.77-36.35 \mathrm{ppm}$. The carbon bonded to the $\mathrm{O}$ atom displayed signals at about $76.45 \mathrm{ppm}$. The other saturated carbons of menthol displayed signals in the region of $16.22-47.06 \mathrm{ppm}$. Their molecular weights and the $\mathrm{C}, \mathrm{H}$, and $\mathrm{N}$ elemental ratios agreed with the results of ESI-MS and elemental analysis, respectively.

\subsection{Antifungal Activity}

The antifungal activities of the target compounds menthol-derived 1,2,4-triazolethioethers $\mathbf{5} \mathbf{a}-\mathbf{5 z}$ were evaluated by the in vitro method against fusarium wilt on cucumber (Fusarium oxysporum f. sp. cucumerinum), speckle on peanut (Cercospora arachidicola), apple ring rot (Physalospora piricola), tomato early blight (Alternaria solani), wheat scab (Gibberella zeae), rice sheath blight (Rhizoeotnia solani), corn southern leaf blight (Bipolaris maydis), and watermelon anthracnose (Colleterichum orbicalare) at $50 \mu \mathrm{g} / \mathrm{mL}$, using the commercial antifungal agent chlorothalonil as positive control. The results are listed in Table 1.

Table 1. Antifungal activity of the target compounds $\mathbf{5 a}-\mathbf{5 z}$ at $50 \mu \mathrm{g} / \mathrm{mL}$.

\begin{tabular}{|c|c|c|c|c|c|c|c|c|}
\hline \multirow{2}{*}{ Compounds } & \multicolumn{8}{|c|}{ Relative Inhibition Rate (\%) against the Fungi } \\
\hline & $\begin{array}{c}\text { F. oxysporum f. } \\
\text { sp. cucumerinum }\end{array}$ & $\begin{array}{c}\text { C. } \\
\text { arachidicola }\end{array}$ & P. piricola & A. solani & G. zeae & R. solani & B. myadis & $\begin{array}{c}\text { C. } \\
\text { orbicalare }\end{array}$ \\
\hline $5 \mathbf{a}(\mathrm{R}=\mathrm{Ph})$ & 22.5 & 48.9 & 45.7 & 45.0 & 22.2 & 22.3 & 32.7 & 30.8 \\
\hline $5 \mathbf{b}\left(\mathrm{R}=o-\mathrm{CH}_{3} \mathrm{Ph}\right)$ & 83.8 & 68.6 & 93.3 & 72.5 & 49.2 & 41.7 & 77.5 & 90.5 \\
\hline $5 c\left(\mathrm{R}=p-\mathrm{CH}_{3} \mathrm{Ph}\right)$ & 24.3 & 47.9 & 54.4 & 35.0 & 29.6 & 14.9 & 27.5 & 24.6 \\
\hline $5 \mathrm{~d}\left(\mathrm{R}=o-\mathrm{OCH}_{3} \mathrm{Ph}\right)$ & 74.3 & 54.8 & 62.8 & 56.9 & 49.2 & 46.6 & 77.5 & 56.3 \\
\hline $5 \mathbf{e}\left(\mathrm{R}=p-\mathrm{OCH}_{3} \mathrm{Ph}\right)$ & 26.7 & 65.2 & 60.0 & 60.0 & 49.2 & 14.9 & 32.5 & 27.1 \\
\hline $5 \mathrm{f}(\mathrm{R}=o-\mathrm{F} \mathrm{Ph})$ & 79.0 & 72.1 & 73.9 & 72.5 & 47.3 & 52.7 & 72.5 & 66.1 \\
\hline $5 \mathrm{~g}(\mathrm{R}=m-\mathrm{F} \mathrm{Ph})$ & 24.3 & 51.4 & 46.1 & 53.8 & 86.5 & 14.9 & 30.0 & 17.3 \\
\hline $5 \mathrm{~h}(\mathrm{R}=p-\mathrm{F} \mathrm{Ph})$ & 22.5 & 21.1 & 17.1 & 30.0 & 23.9 & 16.2 & 32.7 & 35.0 \\
\hline $5 \mathbf{i}(\mathrm{R}=o-\mathrm{Cl} \mathrm{Ph})$ & 76.7 & 79.0 & 79.4 & 69.4 & 62.9 & 41.7 & 70.0 & 73.4 \\
\hline $5 \mathbf{j}(\mathrm{R}=m-\mathrm{Cl} \mathrm{Ph})$ & 45.7 & 58.3 & 62.8 & 50.6 & 49.2 & 13.7 & 55.0 & 39.3 \\
\hline $5 \mathbf{k}(\mathrm{R}=p-\mathrm{Cl} \mathrm{Ph})$ & 14.2 & 21.1 & 17.1 & 45.0 & 23.9 & 16.2 & 14.5 & 26.7 \\
\hline $51(\mathrm{R}=p-\mathrm{Br} \mathrm{Ph})$ & 24.3 & 51.4 & 35.0 & 41.3 & 29.6 & 13.7 & 37.5 & 22.2 \\
\hline $5 \mathrm{~m}(\mathrm{R}=o-\mathrm{I} \mathrm{Ph})$ & 88.6 & 68.6 & 73.9 & 63.1 & 59.0 & 38.0 & 80.0 & 88.0 \\
\hline 5n $(\mathrm{R}=p-\mathrm{I} \mathrm{Ph})$ & 24.3 & 54.8 & 65.6 & 50.6 & 35.5 & 14.9 & 32.5 & 22.2 \\
\hline 5 o $\left(\mathrm{R}=o-\mathrm{CF}_{3} \mathrm{Ph}\right)$ & 64.8 & 79.0 & 65.6 & 66.3 & 59.0 & 39.3 & 67.5 & 66.1 \\
\hline $5 \mathrm{p}\left(\mathrm{R}=m-\mathrm{CF}_{3} \mathrm{Ph}\right)$ & 38.6 & 51.4 & 57.2 & 47.5 & 45.3 & 19.8 & 42.5 & 34.4 \\
\hline $5 \mathrm{q}(\mathrm{R}=o-\mathrm{OH} \mathrm{Ph})$ & 31.4 & 54.8 & 65.6 & 56.9 & 59.0 & 23.4 & 37.5 & 17.3 \\
\hline $5 \mathbf{r}(\mathrm{R}=p-\mathrm{OH} \mathrm{Ph})$ & 18.3 & 32.2 & 24.3 & 40.0 & 32.2 & 55.7 & 28.2 & 22.5 \\
\hline $5 s\left(\mathrm{R}=o-\mathrm{NH}_{2} \mathrm{Ph}\right)$ & 71.9 & 72.1 & 60.0 & 72.5 & 51.2 & 56.3 & 75.0 & 63.7 \\
\hline $5 \mathbf{t}\left(\mathrm{R}=p-\mathrm{NH}_{2} \mathrm{Ph}\right)$ & 76.7 & 68.6 & 62.8 & 56.9 & 53.1 & 55.1 & 55.0 & 56.3 \\
\hline $5 \mathbf{u}\left(\mathrm{R}=p-\mathrm{C}\left(\mathrm{CH}_{3}\right)_{3} \mathrm{Ph}\right)$ & 38.6 & 58.3 & 60.0 & 47.5 & 45.3 & 17.3 & 42.5 & 36.8 \\
\hline $5 \mathbf{v}\left(\mathrm{R}=m, p-\mathrm{OCH}_{3} \mathrm{Ph}\right)$ & 76.7 & 82.4 & 79.4 & 56.9 & 43.3 & 34.4 & 55.0 & 46.6 \\
\hline $5 \mathbf{w}\left(\mathrm{R}=m, m-\mathrm{OCH}_{3} \mathrm{Ph}\right)$ & 50.5 & 61.7 & 73.9 & 53.8 & 51.2 & 35.6 & 57.5 & 56.3 \\
\hline $5 \mathbf{x}(\mathrm{R}=\alpha$-furyl $)$ & 26.7 & 37.6 & 79.4 & 38.1 & 23.7 & 14.9 & 35.0 & 22.2 \\
\hline $5 y(\mathrm{R}=\alpha$-thienyl $)$ & 31.4 & 58.3 & 51.7 & 38.1 & 39.4 & 11.2 & 32.5 & 22.2 \\
\hline $5 \mathbf{z}(\mathrm{R}=\beta$-pyridyl $)$ & 26.7 & 65.2 & 62.8 & 44.4 & 19.8 & 17.3 & 30.0 & 22.2 \\
\hline Chlorothanil & 100 & 73.3 & 75.0 & 73.9 & 73.1 & 96.1 & 90.4 & 91.3 \\
\hline
\end{tabular}

The bioassay results indicated that at the concentration of $50 \mu \mathrm{g} / \mathrm{mL}$, the target compounds $\mathbf{5 a}-\mathbf{5 z}$ exhibited certain antifungal activity against the eight tested fungi, especially against $P$. piricola. Compounds $5 \mathbf{b}\left(\mathrm{R}=o-\mathrm{CH}_{3} \mathrm{Ph}\right), 5 \mathbf{i}(\mathrm{R}=o-\mathrm{Cl} \mathrm{Ph}), \mathbf{5 v}\left(\mathrm{R}=m, p-\mathrm{OCH}_{3} \mathrm{Ph}\right)$ and $5 x(\mathrm{R}=\alpha$-furyl) had inhibition rates of $93.3 \%, 79.4 \%, 79.4 \%$ and $79.4 \%$, respectively, against $P$. piricola, better than that of the positive control chlorothalonil with inhibition rate of $75.0 \%$. Compounds $5 \mathbf{v}\left(\mathrm{R}=m, p-\mathrm{OCH}_{3} \mathrm{Ph}\right), 5 \mathbf{i}(\mathrm{R}=o-\mathrm{Cl} \mathrm{Ph})$ and $5 \mathbf{o}\left(\mathrm{R}=o-\mathrm{CF}_{3} \mathrm{Ph}\right)$ held 
inhibition rates of $82.4 \%, 79.0 \%$ and $79.0 \%$ against $C$. arachidicola, respectively, better than that of the commercial fungicide chlorothalonil with inhibition rate of $73.3 \%$. Compound $5 \mathrm{~g}(\mathrm{R}=m-\mathrm{F} \mathrm{Ph})$ displayed inhibitory activity of $86.5 \%$ against $G$. zeae, better than that of the positive control chlorothalonil with inhibition rate of $73.1 \%$. Compound $5 \mathbf{b}\left(\mathrm{R}=o-\mathrm{CH}_{3} \mathrm{Ph}\right)$ displayed antifungal activity of $90.5 \%$ and $83.8 \%$, respectively, against $C$. orbicalare and $F$. oxysporum f. sp. cucumerinu. Compounds $5 \mathrm{~m}(\mathrm{R}=o$-I $\mathrm{Ph})$ had inhibition rates of $88.6 \%$, $80.0 \%$, and $88.0 \%$, respectively, against F. oxysporum f. sp. cucumerinu, B. myadis and C. orbiculare. In addition, compound $5 \mathbf{b}\left(\mathrm{R}=o-\mathrm{CH}_{3} \mathrm{Ph}\right)$ displayed the best and broad-spectrum antifungal activity against all the tested fungi. Therefore, compound $5 \mathbf{b}\left(\mathrm{R}=o-\mathrm{CH}_{3} \mathrm{Ph}\right)$ deserved further study. To further investigate the structure-activity relationship, 3D-QSAR and molecular docking studies were subsequently performed.

\subsection{CoMFA Analysis}

The 3D-QSAR analysis of the antifungal activity against P. piricola of the target compounds was carried out by the CoMFA method to study the structure-activity relationship of the synthesized compounds. In this work, 23 derivatives were selected to perform 3D-QSAR study and the results of CoMFA model are listed in Table 2. This model with cross-validated coefficient $\left(q^{2}=0.514>0.5\right)$, correlation coefficient $\left(r^{2}=0.991>0.8\right)$, standard error of estimate $(S=0.050)$ and Fischer ratio $(F$ value $=183.384)$ was reliable and effective. In addition, the Scatter plot of predicted ED values vs experimental ED values shown in Figure 1 was drawn by the data presented in Table 3. In Figure 1, it was easily observed that the all points were placed at the nearby of $Y=X$ line.

Table 2. Summary of CoMFA analysis.

\begin{tabular}{cc}
\hline Statistical Parameters & CoMFA \\
\hline$q^{2}$ & 0.514 \\
$r^{2}$ & 0.991 \\
$S$ & 0.050 \\
$F$ & 184.384 \\
Field Contribution (\%) & 75.2 \\
Steric & 24.8 \\
\hline Electrostatic & \\
\hline
\end{tabular}

$q^{2}$ : cross-validated correlational coefficient; $r^{2}$ : non-validated correlational coefficient; $S$ : standard error of estimate; $F$ : the Fischer ratio.

Table 3. The ED values of experimental and predicted activities.

\begin{tabular}{|c|c|c|c|c|c|}
\hline Compound & $\mathbf{R}$ & WM & ED & ED" & Residue \\
\hline $5 a$ & $\mathrm{Ph}$ & 387.20 & -2.66 & -2.61 & -0.05 \\
\hline $5 b$ & $o-\mathrm{CH}_{3} \mathrm{Ph}$ & 401.21 & -1.46 & -1.45 & -0.01 \\
\hline $5 c$ & $p-\mathrm{CH}_{3} \mathrm{Ph}$ & 401.21 & -2.53 & -2.49 & -0.04 \\
\hline $5 d$ & $o-\mathrm{OCH}_{3} \mathrm{Ph}$ & 417.21 & -2.39 & -2.35 & -0.04 \\
\hline $5 e$ & $p-\mathrm{OCH}_{3} \mathrm{Ph}$ & 417.21 & -2.44 & -2.48 & 0.04 \\
\hline $5 f$ & $o-\mathrm{F} \mathrm{Ph}$ & 405.19 & -2.16 & -2.17 & 0.01 \\
\hline $5 g$ & $m-\mathrm{F} \mathrm{Ph}$ & 405.19 & -2.68 & -2.71 & 0.03 \\
\hline $5 \mathrm{~h}$ & $p$-F Ph & 405.19 & -3.29 & -3.34 & 0.04 \\
\hline $5 i$ & $o-\mathrm{Cl} \mathrm{Ph}$ & 421.16 & -2.04 & -2.13 & 0.09 \\
\hline $5 j$ & $m-\mathrm{Cl} \mathrm{Ph}$ & 421.16 & -2.40 & -2.39 & -0.01 \\
\hline $5 \mathrm{k}$ & $p-\mathrm{Cl} \mathrm{Ph}$ & 421.16 & -3.31 & -3.28 & -0.03 \\
\hline 51 & $p$-Br Ph & 465.11 & -2.94 & -2.98 & 0.04 \\
\hline $5 \mathrm{~m}$ & $o-\mathrm{I} \mathrm{Ph}$ & 513.09 & -2.26 & -2.25 & -0.01 \\
\hline $5 n$ & $p$-I Ph & 513.09 & -2.43 & -2.40 & -0.03 \\
\hline 50 & $o-\mathrm{CF}_{3} \mathrm{Ph}$ & 455.19 & -2.38 & -2.38 & 0.00 \\
\hline $5 p$ & $m-\mathrm{CF}_{3} \mathrm{Ph}$ & 455.19 & -2.53 & -2.53 & 0.00 \\
\hline
\end{tabular}


Table 3. Cont.

\begin{tabular}{cccccc}
\hline Compound & $\mathbf{R}$ & $\mathbf{W M}$ & $\mathbf{E D}$ & ED $^{\prime \prime}$ & Residue \\
\hline $\mathbf{5 q}$ & $o-\mathrm{OH} \mathrm{Ph}$ & 403.19 & -2.32 & -2.25 & -0.07 \\
$\mathbf{5} \mathbf{r}$ & $p-\mathrm{OH} \mathrm{Ph}$ & 403.19 & -3.10 & -3.04 & -0.06 \\
$\mathbf{5}$ & $o-\mathrm{NH}_{2} \mathrm{Ph}$ & 402.21 & -2.43 & -2.47 & 0.04 \\
$\mathbf{5}$ & $p-\mathrm{NH}_{2} \mathrm{Ph}$ & 402.21 & -2.38 & -2.44 & 0.06 \\
$\mathbf{5} \mathbf{u}$ & $\left.p-\mathrm{CH}_{3}\right)_{3} \mathrm{Ph}$ & 443.26 & -2.47 & -2.48 & 0.01 \\
$\mathbf{5} \mathbf{v}$ & $m, p-\mathrm{OCH}_{3} \mathrm{Ph}$ & 447.22 & -2.06 & -2.04 & -0.02 \\
$5 \mathbf{w}^{*}$ & $m, m-\mathrm{OCH}_{3} \mathrm{Ph}$ & 447.22 & -2.20 & -2.22 & 0.02 \\
\hline
\end{tabular}

ED: experimental value; ED": predictive value; *: test-set compound.

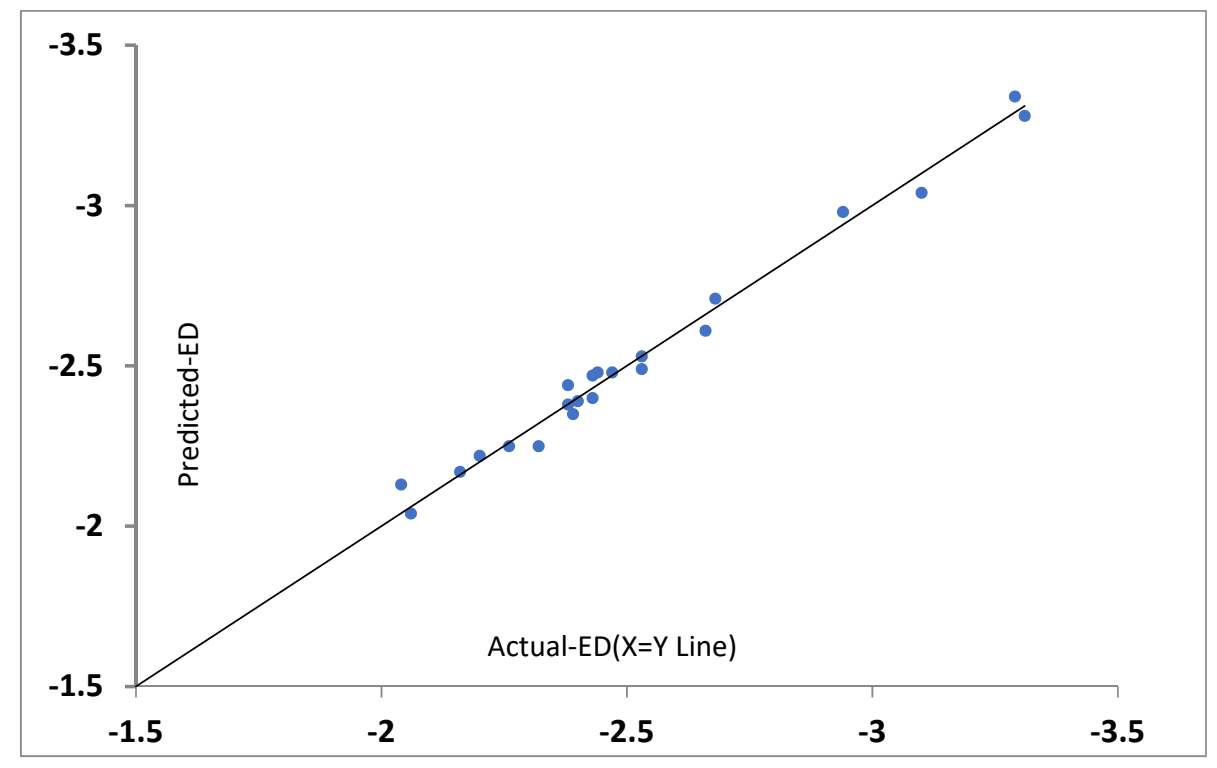

Figure 1. Predicted ED values of CoMFA model vs experimental ED value.

The electrostatic and steric contribution maps of CoMFA are shown in Figure 2. The contribution rate of the electrostatic field and the steric field were $24.8 \%$ and $75.2 \%$, respectively, revealing that the steric field was the major contributor to the increase in the antifungal activity against $P$. piricola. In Figure 2A, there were some green regions located around the 2-position of the benzene ring indicating that the introduction of bulky group in this position was beneficial to increase the antifungal activity. For example, compounds $5 \mathbf{b}\left(\mathrm{R}=o-\mathrm{CH}_{3} \mathrm{Ph}\right), 5 \mathrm{~d}\left(\mathrm{R}=o-\mathrm{OCH}_{3} \mathrm{Ph}\right), 5 \mathrm{f}(\mathrm{R}=o-\mathrm{F} \mathrm{Ph}), 5 \mathbf{i}(\mathrm{R}=o-\mathrm{Cl} \mathrm{Ph}), 5 \mathrm{~m}(\mathrm{R}=o-\mathrm{I} \mathrm{Ph})$, $50\left(\mathrm{R}=0-\mathrm{CF}_{3} \mathrm{Ph}\right), 5 \mathbf{q}(\mathrm{R}=o-\mathrm{OH} \mathrm{Ph})$ and $5 \mathbf{s}\left(\mathrm{R}=o-\mathrm{NH}_{2} \mathrm{Ph}\right)$ displayed higher antifungal activity than compound $5 \mathrm{a}(\mathrm{R}=\mathrm{Ph})$. In contrast, the yellow regions (Figure $2 \mathrm{~A})$ at the 4-position of the phenyl ring, indicated that the introduction of bulky group in this position was associated with worse antifungal activities. For example, compounds $5 \mathrm{c}\left(\mathrm{R}=p-\mathrm{CH}_{3} \mathrm{Ph}\right)$, 5h $(\mathrm{R}=p-\mathrm{F} \mathrm{Ph}), 5 \mathbf{k}(\mathrm{R}=p-\mathrm{Cl} \mathrm{Ph}), 5 \mathbf{l}(\mathrm{R}=p-\mathrm{Br} \mathrm{Ph})$ and $\mathbf{r}(\mathrm{R}=p$ - $\mathrm{OH} \mathrm{Ph})$ displayed worse antifungal activity than compound $5 \mathrm{a}(\mathrm{R}=\mathrm{Ph})$. In Figure $2 \mathrm{~B}$, the electrostatic field contours were displayed in two distinguishing colors. The blue block represents that the introduction of electron donor groups is conducive to enhancing activity and the opposite for the red block. Therefore, the introduction of electron-donating groups at the 4-position and electron-withdrawing groups at the 2-position of benzene rings were both favorable for antifungal activity. For instance, compound $5 \mathbf{c}\left(\mathrm{R}=p-\mathrm{CH}_{3} \mathrm{Ph}\right)$ and $\mathbf{5 e}\left(\mathrm{R}=p-\mathrm{OCH}_{3} \mathrm{Ph}\right)$ exhibited better antifungal activity than that of $5 \mathbf{h}(\mathrm{R}=p-\mathrm{F} \mathrm{Ph}), 5 \mathrm{k}(\mathrm{R}=p-\mathrm{Cl} \mathrm{Ph})$ and $51(\mathrm{R}=p$ - $\mathrm{Br} \mathrm{Ph})$, compounds $5 \mathrm{f}(\mathrm{R}=o-\mathrm{F} \mathrm{Ph}), 5 \mathbf{i}(\mathrm{R}=o-\mathrm{Cl} \mathrm{Ph})$, and $5 \mathrm{~m}(\mathrm{R}=o-\mathrm{I} \mathrm{Ph})$ possessed a higher inhibitory rate than $5 \mathrm{~d}\left(\mathrm{R}=o-\mathrm{OCH}_{3} \mathrm{Ph}\right)$. 


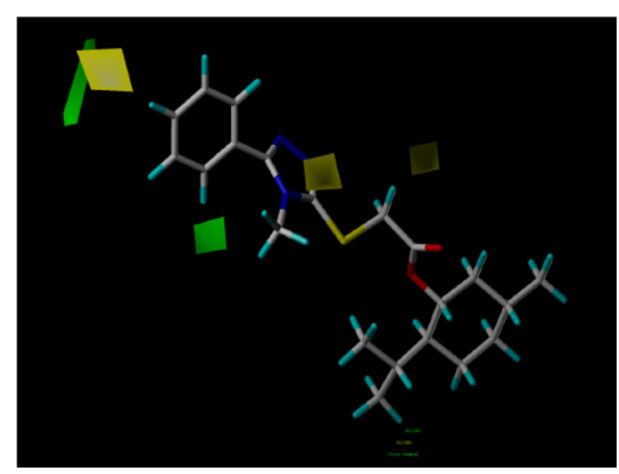

(A)

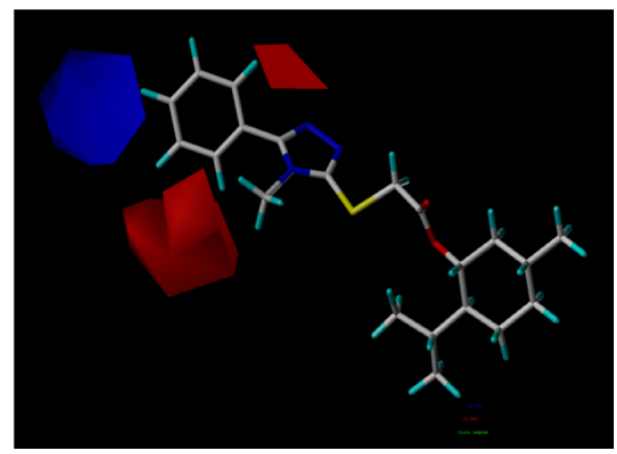

(B)

Figure 2. Contours of CoMFA analysis: (A) contours of steric contribution are represented in yellow and green; (B) contours of electrostatic contribution are represented in red and blue.

\subsection{Molecular Docking Analysis}

For investigating the binding modes of the target compounds in the active site of cytochrome P450 14 $\alpha$-sterol demethylase (CYP51), molecular docking study was carried out using Sybyl-X 2.1.1 software. As a representative sample, the binding modes of compound $5 \mathbf{b}\left(\mathrm{R}=0-\mathrm{CH}_{3} \mathrm{Ph}\right)$ are respectively presented in the $2 \mathrm{D}$ and $3 \mathrm{D}$ pattern (Figure 3 ). It was found that compound $5 \mathbf{b}\left(\mathrm{R}=o-\mathrm{CH}_{3} \mathrm{Ph}\right)$ could readily embed into the binding pocket and interact with the residues around. In particular, the formed hydrogen bond between the $\mathrm{O}$ atom of the ester group of the ligand and the hydroxyl of the residue Tyr76(A) was observed. In addition, the small-molecule ligand also generated hydrophobic interactions with other residues, such as Phe78(A), Met79(A), Leu100(A), and Leu321(A). For comparison, imibenconazole, a commercial CYP51 inhibitor, was also evaluated for its binding modes in the active site, and the obtained results were shown in the 2D pattern (Figure 3D). Most of the residues around compound $5 \mathbf{b}\left(\mathrm{R}=\mathrm{o}-\mathrm{CH}_{3} \mathrm{Ph}\right)$ could be also observed in the case of imibenconazole, revealing that they exhibited similar binding modes in the active site.
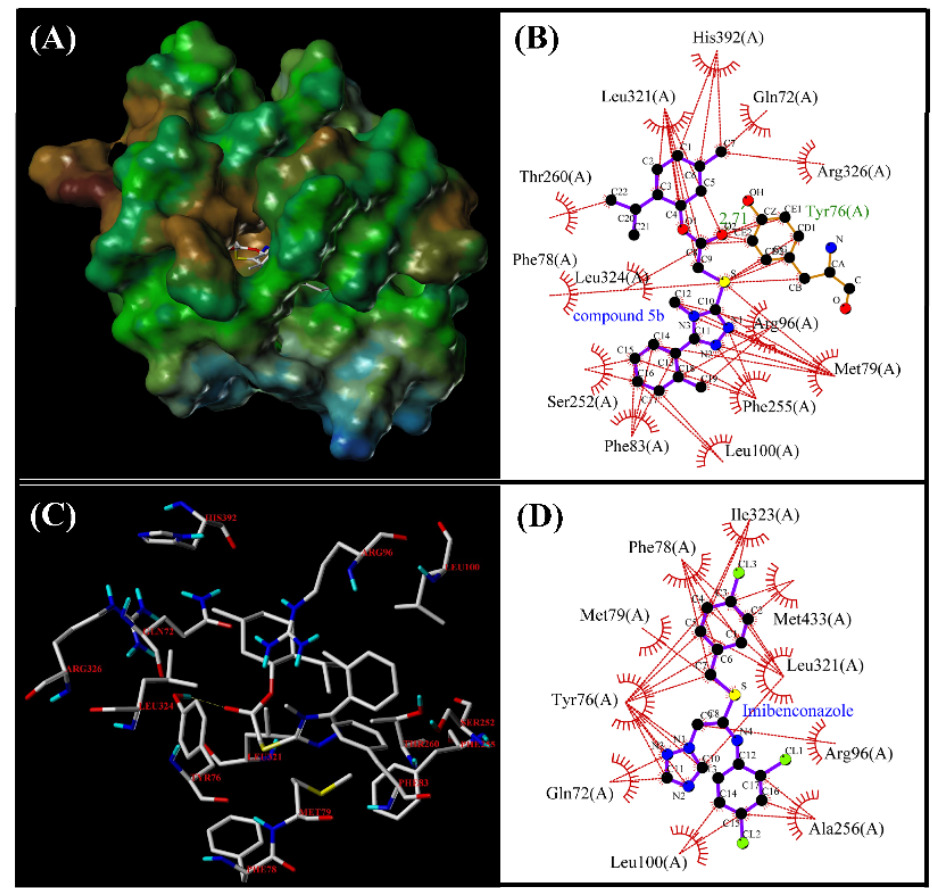

Figure 3. The binding pocket $(A)$ and modes of compound $5 \mathrm{~b}\left(\mathrm{R}=o-\mathrm{CH}_{3} \mathrm{Ph}\right)$ respectively presented in the $2 \mathrm{D}(\mathrm{B})$ and $3 \mathrm{D}$ pattern $(\mathrm{C})$, along with the binding modes of imibenconazole presented in the 2D pattern $(\mathbf{D})$. 
Moreover, their binding affinity with the target protein could be expressed by total score [38], which was automatically generated from the docking process. To our delight, all of the total scores of the target compounds (6.5160-9.1989) were higher than that of imibenconazole (5.4657), indicating that the target compounds showed stronger binding affinity with CYP51 than imibenconazole.

\section{Experimental Section}

3.1. General Information

The GC analysis was conducted on an Agilent 6890 GC (Agilent Technologies Inc., Santa Clara, CA, USA) equipped with column HP-1 $(30 \mathrm{~m}, 0.530 \mathrm{~mm}, 0.88 \mu \mathrm{m})$. The IR spectra were recorded on a Nicolet iS50 FT-IR spectrometer (Thermo Scientific Co., Ltd., Madison, WI, USA) using the KBr pellet method. The HPLC analysis was conducted on Waters 1525 HPLC instrument (Waters Co., Ltd., Milford, MA, USA) equipped with column C18 $5 \mu \mathrm{m}(4.6 \mathrm{~mm} \times 150 \mathrm{~mm})$ and Waters 2998 PDA detector. The melting points were recorded using an MP420 automatic melting point apparatus (Hanon Instruments Co., Ltd., Jinan, China) and were not corrected. The ${ }^{1} \mathrm{H}$ NMR and ${ }^{13} \mathrm{C}$ NMR spectra were determined on a Bruker Avance III HD $500 \mathrm{MHz} / 600 \mathrm{MHz}$ spectrometer (Switzerland Bruker Co., Ltd., Zurich, Switzerland) using $\mathrm{CDCl}_{3}$ as the solvent and TMS as an internal standard. Mass spectra were obtained by means of the electrospray ionization (ESI) method on the TSQ Quantum Access MAX HPLC-MS instrument (Thermo Scientific Co., Ltd., Waltham, MA, USA). Elemental analyses were measured using a PE 2400 II elemental analyzer (Perkin-Elmer Instruments Co., Ltd., Waltham, MA, USA). L-menthol (GC purity 99\%) was provided by Shanghai Macklin Biochemical Co., Ltd. (Shanghai, China). A series of substituted aryl hydrazides were purchased from Shanghai Aladdin Biochemical Technology Co., Ltd. (Shanghai, China). Other reagents were purchased from commercial suppliers and used as received.

\subsection{Synthesis of (-)-Menthyl-2-chloroacetate (2)}

A solution of chloroacetyl chloride $(12.42 \mathrm{~g}, 0.11 \mathrm{~mol})$ in dry DCM $(20 \mathrm{~mL})$ was added slowly to a mixture of (-)-menthol $(15.63 \mathrm{~g}, 0.10 \mathrm{~mol})$, triethylamine $(11.13 \mathrm{~g}, 0.11 \mathrm{~mol})$ and DMAP $(0.80 \mathrm{~g}, 0.006 \mathrm{~mol})$ in dry DCM $(15 \mathrm{~mL})$ with ice-bath cooling. The reaction process was monitored by TLC. Upon completion, saturated aqueous $\mathrm{NaHCO}_{3}(5 \mathrm{~mL})$ was added to destroy the unreacted acyl chloride. Then, the organic layer was separated, washed with deionized water three times, dried over $\mathrm{Na}_{2} \mathrm{SO}_{4}$, and concentrated under reduced pressure. The crude product was further purified by silica gel chromatography (petroleum ether/ethyl acetate $10: 1, \mathrm{v} / \mathrm{v}$ ) to obtain the intermediate 2 as a colorless transparent liquid. Yield 92\%; ${ }^{1} \mathrm{H}$ NMR $\left(600 \mathrm{MHz}, \mathrm{CDCl}_{3}\right) \delta 4.73(\mathrm{td}, J=10.9,4.4 \mathrm{~Hz}, 1 \mathrm{H}), 4.10(\mathrm{~s}, 2 \mathrm{H}), 2.00$ $(\mathrm{dt}, J=12.1,4.1 \mathrm{~Hz}, 1 \mathrm{H}), 1.85(\mathrm{pt}, J=7.0,3.5 \mathrm{~Hz}, 1 \mathrm{H}), 1.69(\mathrm{dt}, J=13.9,3.4 \mathrm{~Hz}, 2 \mathrm{H}), 1.48$ (dtd, $J=12.1,6.7,6.1,3.2 \mathrm{~Hz}, 1 \mathrm{H}), 1.40(\mathrm{~s}, 1 \mathrm{H}), 1.07-0.98(\mathrm{~m}, 2 \mathrm{H}), 0.92-0.88(\mathrm{~m}, 6 \mathrm{H}), 0.85$ $(\mathrm{dd}, J=12.3,3.6 \mathrm{~Hz}, 1 \mathrm{H}), 0.75(\mathrm{~d}, J=7.0 \mathrm{~Hz}, 3 \mathrm{H}) ;{ }^{13} \mathrm{C} \mathrm{NMR}\left(151 \mathrm{MHz}, \mathrm{CDCl}_{3}\right) \delta 168.07$, $76.35,46.93,40.63,36.03,34.11,31.41,26.18,23.36,21.97,20.75,16.28$. These data were in agreement with that of the literature [39].

\subsection{General Procedure of Menthol-Based 1,2,4-Triazole-thioether Compounds (5a-5w)}

Intermediate 4 was prepared according to a method described in the literature [40]. The intermediate $4(2.0 \mathrm{mmol})$ and sodium acetate trihydrate $(0.27 \mathrm{~g}, 2.0 \mathrm{mmol})$ were dissolved in $15 \mathrm{~mL}$ mixed solvent of ethanol and water (ethanol/water 2:1, v/v). Then, the mixture was stirred at $45^{\circ} \mathrm{C}$ for $2 \mathrm{~h}$. Afterwards, a solution of menthyl chloroacetate $2(0.46 \mathrm{~g}, 2.0 \mathrm{mmol})$ in $5 \mathrm{~mL}$ EtOH was slowly added to the mixture and continuously refluxed at $80{ }^{\circ} \mathrm{C}$ for $6 \mathrm{~h}$. Upon completion of the reaction, the mixture was concentrated in vacuum. Then, the crude product was poured into saturated sodium bicarbonate solution, and the mixture was extracted with dichloromethane $(3 \times 20 \mathrm{~mL})$. The organic layer was washed with saturated $\mathrm{NaCl}$ solution three times, dried over anhydrous sodium sulfate, 
and purified by silica gel column chromatography (petroleum ether/ethyl acetate 3:1, $v / v$ ) to afford the target compounds $5 \mathrm{a}-5 \mathrm{w}$ in yields of $50 \%$ to $76 \%$.

Compound (5a): (1R,2S,5R)-2-isopropyl-5-methylcyclohexyl-2-((4-methyl-5-phenyl-4H1,2,4-triazol-3-yl) thio)acetate. White solid. Yield: $63.0 \%$, m.p. $124.7-125.1{ }^{\circ} \mathrm{C}$; IR (KBr, $\mathrm{cm}^{-1}$ ): 2960, 2934, 2915, 2864 (s, C-H), 1735(s, C=O), 1482 (s, C=N), 703 (w, C-S-C); ${ }^{1} \mathrm{H}$ NMR $\left(600 \mathrm{MHz}, \mathrm{CDCl}_{3}\right) \delta 7.62\left(\mathrm{dd}, J=6.6,3.0 \mathrm{~Hz}, 2 \mathrm{H}, \mathrm{C}_{16}-\mathrm{H}, \mathrm{C}_{20}-\mathrm{H}\right), 7.54-7.46(\mathrm{~m}, 3 \mathrm{H}$, $\left.\mathrm{C}_{17}-\mathrm{H}, \mathrm{C}_{18}-\mathrm{H}, \mathrm{C}_{19}-\mathrm{H}\right), 4.71\left(\mathrm{td}, J=10.9,4.5 \mathrm{~Hz}, 1 \mathrm{H}, \mathrm{C}_{1}-\mathrm{H}\right), 4.08\left(\mathrm{~s}, 2 \mathrm{H}, \mathrm{C}_{12}-\mathrm{H}\right), 3.66(\mathrm{~s}, 3 \mathrm{H}$, $\left.\mathrm{C}_{21}-\mathrm{H}\right), 1.98\left(\mathrm{~d}, \mathrm{~J}=12.6 \mathrm{~Hz}, 1 \mathrm{H}, \mathrm{C}_{6}-\mathrm{Ha}\right), 1.86-1.79\left(\mathrm{~m}, 1 \mathrm{H}, \mathrm{C}_{7}-\mathrm{H}\right), 1.69-1.64\left(\mathrm{~m}, 2 \mathrm{H}, \mathrm{C}_{4}-\mathrm{Ha}\right.$, $\left.\mathrm{C}_{3}-\mathrm{Ha}\right), 1.45\left(\mathrm{ddt}, J=8.8,5.6,2.6 \mathrm{~Hz}, 1 \mathrm{H}, \mathrm{C}_{5}-\mathrm{H}\right), 1.38\left(\mathrm{t}, J=11.7 \mathrm{~Hz}, 1 \mathrm{H}, \mathrm{C}_{2}-\mathrm{H}\right), 1.06-0.95(\mathrm{~m}$, $\left.2 \mathrm{H}, \mathrm{C}_{6}-\mathrm{H}_{\mathrm{b}}, \mathrm{C}_{3}-\mathrm{H}_{\mathrm{b}}\right), 0.88\left(\mathrm{dd}, J=8.7,6.7 \mathrm{~Hz}, 6 \mathrm{H}, \mathrm{C}_{10}-\mathrm{H}, \mathrm{C}_{8}-\mathrm{H}\right.$ or $\left.\mathrm{C}_{9}-\mathrm{H}\right), 0.83(\mathrm{dd}, J=12.7$, $\left.3.4 \mathrm{~Hz}, 1 \mathrm{H}, \mathrm{C}_{4}-\mathrm{H}_{\mathrm{b}}\right), 0.73\left(\mathrm{~d}, \mathrm{~J}=7.0 \mathrm{~Hz}, 3 \mathrm{H}, \mathrm{C}_{8}-\mathrm{H}\right.$ or $\left.\mathrm{C}_{9}-\mathrm{H}\right) ;{ }^{13} \mathrm{C} \mathrm{NMR}\left(150 \mathrm{MHz} \mathrm{CDCl}_{3}\right) \delta$ $168.07,156.10,150.54,130.15,128.92,128.62,126.99,76.35,46.93,40.63,36.03,34.11,31.86$, 31.41, 26.18, 23.36, 21.97, 20.75, 16.28; ESI-MS $m / z: 388.20[\mathrm{M}+\mathrm{H}]^{+}$. Anal. Calcd. for $\mathrm{C}_{21} \mathrm{H}_{29} \mathrm{~N}_{3} \mathrm{O}_{2} \mathrm{~S}: \mathrm{C}, 65.08 ; \mathrm{H}, 7.54 ; \mathrm{N}, 10.84$; Found: C, 65.06; H, 7.52; N, 10.37 .

Compound (5b): (1R,2S,5R)-2-isopropyl-5-methylcyclohexyl-2-((4-methyl-5-(o-tolyl)-4H1,2,4-triazol-3-yl)thio)acetate. Pale yellow liquid. Yield: $58.3 \%$, IR (KBr, $\left.\mathrm{cm}^{-1}\right)$ : 2954, 2927, 2869 (s, C-H), 1731(s, C=O), 1457 (s, C=N), 706 (w, C-S-C); ${ }^{1} \mathrm{H} \mathrm{NMR} \mathrm{(500} \mathrm{MHz,} \mathrm{CDCl} 3$ ) $\delta$ $7.42\left(\mathrm{ddd}, J=8.5,6.0,2.7 \mathrm{~Hz}, 1 \mathrm{H}, \mathrm{C}_{20}-\mathrm{H}\right), 7.35\left(\mathrm{~d}, J=7.6 \mathrm{~Hz}, 1 \mathrm{H}, \mathrm{C}_{19}-\mathrm{H}\right), 7.32-7.29(\mathrm{~m}, 2 \mathrm{H}$, $\left.\mathrm{C}_{18}-\mathrm{H}, \mathrm{C}_{17}-\mathrm{H}\right), 4.75\left(\mathrm{td}, J=10.9,4.4 \mathrm{~Hz}, 1 \mathrm{H}, \mathrm{C}_{1}-\mathrm{H}\right), 4.12\left(\mathrm{~s}, 2 \mathrm{H}, \mathrm{C}_{12}-\mathrm{H}\right), 3.44\left(\mathrm{~s}, 3 \mathrm{H}, \mathrm{C}_{21}-\mathrm{H}\right)$, $2.25\left(\mathrm{~s}, 3 \mathrm{H}, \mathrm{C}_{22}-\mathrm{H}\right), 2.01\left(\mathrm{dtd}, J=12.0,4.1,1.8 \mathrm{~Hz}, 1 \mathrm{H}, \mathrm{C}_{6}-\mathrm{Ha}\right), 1.90\left(\mathrm{~d}, J=4.1 \mathrm{~Hz}, 1 \mathrm{H}, \mathrm{C}_{7}-\mathrm{H}\right)$, $1.71\left(\mathrm{t}, J=2.6 \mathrm{~Hz}, 1 \mathrm{H}, \mathrm{C}_{4}-\mathrm{Ha}\right), 1.68\left(\mathrm{q}, J=2.8 \mathrm{~Hz}, 1 \mathrm{H}, \mathrm{C}_{3}-\mathrm{Ha}\right), 1.49$ (dddd, $J=12.1,8.9,6.3,3.2$ $\left.\mathrm{Hz}, 1 \mathrm{H}, \mathrm{C}_{5}-\mathrm{H}\right), 1.45-1.38\left(\mathrm{~m}, 1 \mathrm{H}, \mathrm{C}_{2}-\mathrm{H}\right), 1.11-1.04\left(\mathrm{~m}, 1 \mathrm{H}, \mathrm{C}_{3}-\mathrm{H}_{\mathrm{b}}\right), 1.04-0.99\left(\mathrm{~m}, 1 \mathrm{H}, \mathrm{C}_{6}-\mathrm{H}_{\mathrm{b}}\right)$, $0.92\left(\mathrm{~d}, J=4.6 \mathrm{~Hz}, 3 \mathrm{H}, \mathrm{C}_{10}-\mathrm{H}\right), 0.91\left(\mathrm{~d}, J=5.1 \mathrm{~Hz}, 3 \mathrm{H}, \mathrm{C}_{8}-\mathrm{H}\right.$ or $\left.\mathrm{C}_{9}-\mathrm{H}\right), 0.88(\mathrm{~d}, J=9.6 \mathrm{~Hz}, 1 \mathrm{H}$, $\left.\mathrm{C}_{4}-\mathrm{H}_{\mathrm{b}}\right), 0.76\left(\mathrm{~d}, \mathrm{~J}=7.0 \mathrm{~Hz}, 3 \mathrm{H}, \mathrm{C}_{8}-\mathrm{H}\right.$ or $\left.\mathrm{C}_{9}-\mathrm{H}\right) ;{ }^{13} \mathrm{C} \mathrm{NMR}\left(125 \mathrm{MHz}, \mathrm{CDCl}_{3}\right) \delta 168.21,155.91$, $149.79,138.45,130.79,130.54,130.34,126.57,126.11,76.47,47.05,40.76,36.05,34.23,31.53$, 31.00, 26.32, 23.49, 22.09, 20.86, 19.82, 16.41; ESI-MS m/z: $402.22[\mathrm{M}+\mathrm{H}]^{+}$. Anal. Calcd. for $\mathrm{C}_{22} \mathrm{H}_{31} \mathrm{~N}_{3} \mathrm{O}_{2} \mathrm{~S}: \mathrm{C}, 65.80 ; \mathrm{H}, 7.78 ; \mathrm{N}, 10.46$; Found: C, 65.72; H, 7.73; N, 10.40 .

Compound (5c): (1R,2S,5R)-2-isopropyl-5-methylcyclohexyl-2-((4-methyl-5-(p-tolyl)-4H1,2,4-triazol-3-yl)thio)acetate. White solid. Yield: 70.0\%, m.p. $124.0-125.2{ }^{\circ} \mathrm{C} ; \mathrm{IR}\left(\mathrm{KBr}, \mathrm{cm}^{-1}\right)$ : 2947, 2935, 2917, 2865(s, C-H), 1735(s, C=O), 1475 (s, C=N), 707 (w, C-S-C); ${ }^{1} \mathrm{H}$ NMR $\left(500 \mathrm{MHz}, \mathrm{CDCl}_{3}\right) \delta 7.51\left(\mathrm{~d}, J=8.2 \mathrm{~Hz}, 2 \mathrm{H}, \mathrm{C}_{15}-\mathrm{H}, \mathrm{C}_{20}-\mathrm{H}\right), 7.30\left(\mathrm{~d}, J=7.9 \mathrm{~Hz}, 2 \mathrm{H}, \mathrm{C}_{17}-\mathrm{H}\right.$, $\left.\mathrm{C}_{19}-\mathrm{H}\right), 4.71\left(\mathrm{td}, \mathrm{J}=10.9,4.4 \mathrm{~Hz}, 1 \mathrm{H}, \mathrm{C}_{1}-\mathrm{H}\right), 4.07\left(\mathrm{~s}, 2 \mathrm{H}, \mathrm{C}_{12}-\mathrm{H}\right), 3.65\left(\mathrm{~s}, 3 \mathrm{H}, \mathrm{C}_{21}-\mathrm{H}\right), 2.42(\mathrm{~s}$, $\left.3 \mathrm{H}, \mathrm{C}_{22}-\mathrm{H}\right), 1.98\left(\mathrm{dtd}, J=12.1,4.1,1.8 \mathrm{~Hz}, 1 \mathrm{H}, \mathrm{C}_{6}-\mathrm{Ha}\right), 1.82\left(\mathrm{dt}, J=6.9,3.5 \mathrm{~Hz}, 1 \mathrm{H}, \mathrm{C}_{7}-\mathrm{H}\right)$, $1.68\left(\mathrm{~h}, J=2.1 \mathrm{~Hz}, 1 \mathrm{H}, \mathrm{C}_{4}-\mathrm{Ha}\right), 1.65$ (q, $\left.J=2.9 \mathrm{~Hz}, 1 \mathrm{H}, \mathrm{C}_{3}-\mathrm{Ha}\right), 1.45$ (dddt, $J=12.3,9.6,6.6$, $\left.3.3 \mathrm{~Hz}, 1 \mathrm{H}, \mathrm{C}_{5}-\mathrm{H}\right), 1.38\left(\mathrm{ddt}, J=14.1,10.9,3.2 \mathrm{~Hz}, 1 \mathrm{H}, \mathrm{C}_{2}-\mathrm{H}\right), 1.08-1.01\left(\mathrm{~m}, 1 \mathrm{H}, \mathrm{C}_{3}-\mathrm{H}_{\mathrm{b}}\right)$, $1.01-0.95\left(\mathrm{~m}, 1 \mathrm{H}, \mathrm{C}_{6}-\mathrm{H}_{\mathrm{b}}\right), 0.89\left(\mathrm{~d}, J=6.6 \mathrm{~Hz}, 3 \mathrm{H}, \mathrm{C}_{10}-\mathrm{H}\right), 0.87\left(\mathrm{~d}, J=7.1 \mathrm{~Hz}, 3 \mathrm{H}, \mathrm{C}_{8}-\mathrm{H}\right.$ or $\left.\mathrm{C}_{9}-\mathrm{H}\right), 0.86-0.81\left(\mathrm{~m}, 1 \mathrm{H}, \mathrm{C}_{4}-\mathrm{H}_{\mathrm{b}}\right), 0.72\left(\mathrm{~d}, \mathrm{~J}=6.9 \mathrm{~Hz}, 3 \mathrm{H}, \mathrm{C}_{8}-\mathrm{H}\right.$ or $\left.\mathrm{C}_{9}-\mathrm{H}\right) ;{ }^{13} \mathrm{C}$ NMR $(125 \mathrm{MHz}$, $\left.\mathrm{CDCl}_{3}\right) \delta 168.11,156.18,150.29,140.35,129.59,128.49,124.06,76.33,46.92,40.63,36.06,34.11$, 31.83, 31.41, 26.17, 23.36, 21.97, 21.44, 20.75, 16.27; ESI-MS m/z: $402.21[\mathrm{M}+\mathrm{H}]^{+}$. Anal. Calcd. for $\mathrm{C}_{22} \mathrm{H}_{31} \mathrm{~N}_{3} \mathrm{O}_{2} \mathrm{~S}$ : C, 65.80; H, 7.78; N,10.46; Found: C, 65.70; H, 7.72; N, 10.42 .

Compound (5d): (1R,2S,5R)-2-isopropyl-5-methylcyclohexyl-2-((5-(2-methoxyphenyl)-4methyl-4H-1,2,4-triazol-3-yl)thio)acetate. Pale yellow liquid. Yield: $68.2 \%, \mathrm{IR}\left(\mathrm{KBr}, \mathrm{cm}^{-1}\right)$ : 2954, 2928, 2869 (s, C-H), 1731(s, C=O), 1476 (s, C=N), 700 (w, C-S-C); ${ }^{1} \mathrm{H}$ NMR (500 MHz, $\left.\mathrm{CDCl}_{3}\right) \delta 7.51-7.45\left(\mathrm{~m}, 2 \mathrm{H}, \mathrm{C}_{20}-\mathrm{H}, \mathrm{C}_{18}-\mathrm{H}\right), 7.07\left(\mathrm{t}, J=7.5 \mathrm{~Hz}, 1 \mathrm{H}, \mathrm{C}_{19}-\mathrm{H}\right), 6.99(\mathrm{~d}, J=8.7 \mathrm{~Hz}$, $\left.1 \mathrm{H}, \mathrm{C}_{17}-\mathrm{H}\right), 4.72\left(\mathrm{td}, J=10.9,4.4 \mathrm{~Hz}, 1 \mathrm{H}, \mathrm{C}_{1}-\mathrm{H}\right), 4.10\left(\mathrm{~s}, 2 \mathrm{H}, \mathrm{C}_{12}-\mathrm{H}\right), 3.81\left(\mathrm{~s}, 3 \mathrm{H}, \mathrm{C}_{22}-\mathrm{H}\right)$, $3.44\left(\mathrm{~s}, 3 \mathrm{H}, \mathrm{C}_{21}-\mathrm{H}\right), 2.02-1.96\left(\mathrm{~m}, 1 \mathrm{H}, \mathrm{C}_{6}-\mathrm{Ha}\right), 1.86\left(\mathrm{dd}, J=7.0,4.2 \mathrm{~Hz}, 1 \mathrm{H}, \mathrm{C}_{7}-\mathrm{H}\right), 1.68(\mathrm{q}$, $\left.J=2.8,2.2 \mathrm{~Hz}, 1 \mathrm{H}, \mathrm{C}_{4}-\mathrm{Ha}\right), 1.65\left(\mathrm{q}, J=2.8 \mathrm{~Hz}, 1 \mathrm{H}, \mathrm{C}_{3}-\mathrm{Ha}\right), 1.47$ (dddq, $J=15.3,8.9,6.2$, $\left.3.3 \mathrm{~Hz}, 1 \mathrm{H}, \mathrm{C}_{5}-\mathrm{H}\right), 1.42-1.35\left(\mathrm{~m}, 1 \mathrm{H}, \mathrm{C}_{2}-\mathrm{H}\right), 1.09-1.02\left(\mathrm{~m}, 1 \mathrm{H}, \mathrm{C}_{3}-\mathrm{H}_{\mathrm{b}}\right), 1.01-0.96(\mathrm{~m}, 1 \mathrm{H}$, $\left.\mathrm{C}_{6}-\mathrm{H}_{\mathrm{b}}\right), 0.89\left(\mathrm{t}, \mathrm{J}=6.6 \mathrm{~Hz}, 6 \mathrm{H}, \mathrm{C}_{10}-\mathrm{H}, \mathrm{C}_{8}-\mathrm{H}\right.$ or $\left.\mathrm{C}_{9}-\mathrm{H}\right), 0.83\left(\mathrm{~m}, 1 \mathrm{H}, \mathrm{C}_{4}-\mathrm{H}_{\mathrm{b}}\right), 0.74(\mathrm{~d}, \mathrm{~J}=7.0 \mathrm{~Hz}$, $3 \mathrm{H}, \mathrm{C}_{8}-\mathrm{H}$ or $\left.\mathrm{C}_{9}-\mathrm{H}\right) ;{ }^{13} \mathrm{C}$ NMR $\left(125 \mathrm{MHz} \mathrm{CDCl}_{3}\right) \delta 168.14,157.23,154.60,149.85,132.27$, $132.15,121.12,116.15,111.07,76.30,55.55,46.93,40.63,35.86,34.13,31.42,31.16,26.18,23.38$, 21.98, 20.74, 16.28; ESI-MS m/z: $418.22[\mathrm{M}+\mathrm{H}]^{+}$. Anal. Calcd. for $\mathrm{C}_{22} \mathrm{H}_{31} \mathrm{~N}_{3} \mathrm{O}_{3} \mathrm{~S}$ : C, 63.28; H, 7.48; N,10.06; Found: C, 63.25; H, 7.45; N, 10.05 . 
Compound (5e): (1R,2S,5R)-2-isopropyl-5-methylcyclohexyl-2-((5-(4-methoxyphenyl)-4methyl-4H-1,2,4-triazol-3-yl)thio)acetate. White solid. Yield: $66.8 \%$, m.p. $106.9-108.4{ }^{\circ} \mathrm{C}$; IR $\left(\mathrm{KBr}, \mathrm{cm}^{-1}\right)$ : 2952, 2936, 2869 (s, C-H), 1735(s, C=O), 1477 (s, C=N), 714 (w, C-S-C); ${ }^{1} \mathrm{H}$ NMR $\left(500 \mathrm{MHz} \mathrm{CDCl}_{3}\right) \delta 7.56\left(\mathrm{~d}, \mathrm{~J}=8.8 \mathrm{~Hz}, 2 \mathrm{H}, \mathrm{C}_{16}-\mathrm{H}, \mathrm{C}_{20}-\mathrm{H}\right), 7.00\left(\mathrm{~d}, \mathrm{~J}=8.7 \mathrm{~Hz}, 2 \mathrm{H}, \mathrm{C}_{17}-\mathrm{H}\right.$, $\left.\mathrm{C}_{19}-\mathrm{H}\right), 4.70\left(\mathrm{td}, \mathrm{J}=10.9,4.4 \mathrm{~Hz}, 1 \mathrm{H}, \mathrm{C}_{1}-\mathrm{H}\right), 4.06\left(\mathrm{~s}, 2 \mathrm{H}, \mathrm{C}_{12}-\mathrm{H}\right), 3.85\left(\mathrm{~s}, 3 \mathrm{H}, \mathrm{C}_{22}-\mathrm{H}\right), 3.64(\mathrm{~s}$, $\left.3 \mathrm{H}, \mathrm{C}_{21}-\mathrm{H}\right), 2.00-1.95\left(\mathrm{~m}, 1 \mathrm{H}, \mathrm{C}_{6}-\mathrm{Ha}\right), 1.82$ (qt, $\left.J=7.0,3.5 \mathrm{~Hz}, 1 \mathrm{H}, \mathrm{C}_{7}-\mathrm{H}\right), 1.67$ (q, J = 2.5, $\left.1.8 \mathrm{~Hz}, 1 \mathrm{H}, \mathrm{C}_{4}-\mathrm{Ha}\right), 1.64\left(\mathrm{q}, J=2.9 \mathrm{~Hz}, 1 \mathrm{H}, \mathrm{C}_{3}-\mathrm{Ha}\right), 1.46(\mathrm{dtd}, J=12.1,5.9,5.5,3.0 \mathrm{~Hz}, 1 \mathrm{H}$, $\left.\mathrm{C}_{5}-\mathrm{H}\right), 1.42-1.35\left(\mathrm{~m}, 1 \mathrm{H}, \mathrm{C}_{2}-\mathrm{H}\right), 1.08-1.00\left(\mathrm{~m}, 1 \mathrm{H}, \mathrm{C}_{3}-\mathrm{H}_{\mathrm{b}}\right), 1.00-0.93\left(\mathrm{~m}, 1 \mathrm{H}, \mathrm{C}_{6}-\mathrm{H}_{\mathrm{b}}\right), 0.88$ $\left(\mathrm{d}, J=6.8 \mathrm{~Hz}, 3 \mathrm{H}, \mathrm{C}_{10}-\mathrm{H}\right), 0.87\left(\mathrm{~d}, J=7.7 \mathrm{~Hz}, 3 \mathrm{H}, \mathrm{C}_{8}-\mathrm{H}\right.$ or $\left.\mathrm{C}_{9}-\mathrm{H}\right), 0.85-0.80\left(\mathrm{~m}, 1 \mathrm{H}, \mathrm{C}_{4}-\mathrm{H}_{\mathrm{b}}\right)$, $0.72\left(\mathrm{~d}, J=6.9 \mathrm{~Hz}, 3 \mathrm{H}, \mathrm{C}_{8}-\mathrm{H}\right.$ or $\left.\mathrm{C}_{9}-\mathrm{H}\right) ;{ }^{13} \mathrm{C} \mathrm{NMR}\left(125 \mathrm{MHz}, \mathrm{CDCl}_{3}\right) \delta 168.13,161.01,156.00$, 150.12, 130.06, 119.26, 114.36, 76.33, 55.41, 46.92, 40.63, 36.09, 34.11, 31.83, 31.41, 26.17, 23.35, 21.97, 20.75, 16.27; ESI-MS $m / z: 418.23[\mathrm{M}+\mathrm{H}]^{+}$. Anal. Calcd. for $\mathrm{C}_{22} \mathrm{H}_{31} \mathrm{~N}_{3} \mathrm{O}_{3} \mathrm{~S}: \mathrm{C}, 63.28$; H, 7.48; N,10.06; Found: C, 63.23; H, 7.43; N, 10.02 .

Compound (5f): (1R,2S,5R)-2-isopropyl-5-methylcyclohexyl-2-((5-(2-fluorophenyl)-4-methyl4H-1,2,4-triazol-3-yl)thio) acetate. Pale yellow liquid. Yield: $62.8 \%$, IR $\left(\mathrm{KBr}_{\mathrm{cm}} \mathrm{cm}^{-1}\right): 2955,2928$, 2869 (s, C-H), 1732(s, C=O), 1474 (s, C=N), 671 (w, C-S-C); ${ }^{1} \mathrm{H}$ NMR (500 MHz, CDCl ${ }_{3}$ ) $\delta 7.63\left(\mathrm{td}, J=7.4,1.8 \mathrm{~Hz}, 1 \mathrm{H}, \mathrm{C}_{20}-\mathrm{H}\right), 7.52\left(\mathrm{tdd}, J=7.5,5.3,1.8 \mathrm{~Hz}, 1 \mathrm{H}, \mathrm{C}_{18}-\mathrm{H}\right), 7.30(\mathrm{t}$, $\left.J=7.5 \mathrm{~Hz}, 1 \mathrm{H}, \mathrm{C}_{19}-\mathrm{H}\right), 7.24-7.18\left(\mathrm{~m}, 1 \mathrm{H}, \mathrm{C}_{17}-\mathrm{H}\right), 4.72\left(\mathrm{td}, J=10.9,4.4 \mathrm{~Hz}, 1 \mathrm{H}, \mathrm{C}_{1}-\mathrm{H}\right), 4.10$ (s, 2H, C $12-\mathrm{H}), 3.55\left(\mathrm{~d}, J=2.4 \mathrm{~Hz}, 3 \mathrm{H}, \mathrm{C}_{21}-\mathrm{H}\right), 1.99\left(\mathrm{~d}, J=12.0 \mathrm{~Hz}, 1 \mathrm{H}, \mathrm{C}_{6}-\mathrm{H}_{\mathrm{a}}\right), 1.85$ (qd, $\left.J=7.0,2.7 \mathrm{~Hz}, 1 \mathrm{H}, \mathrm{C}_{7}-\mathrm{H}\right), 1.68\left(\mathrm{q}, J=2.8,2.3 \mathrm{~Hz}, 1 \mathrm{H}, \mathrm{C}_{4}-\mathrm{H}_{\mathrm{a}}\right), 1.65\left(\mathrm{q}, J=2.8 \mathrm{~Hz}, 1 \mathrm{H}, \mathrm{C}_{3}-\mathrm{H}_{\mathrm{a}}\right)$, 1.46 (dddq, $\left.J=12.3,9.5,6.5,3.3 \mathrm{~Hz}, 1 \mathrm{H}, \mathrm{C}_{5}-\mathrm{H}\right), 1.38\left(\mathrm{ddt}, J=14.2,11.0,3.1 \mathrm{~Hz}, 1 \mathrm{H}, \mathrm{C}_{2}-\mathrm{H}\right)$, 1.08-1.01 (m, 1H, $\left.\mathrm{C}_{3}-\mathrm{H}_{\mathrm{b}}\right), 1.01-0.95\left(\mathrm{~m}, 1 \mathrm{H}, \mathrm{C}_{6}-\mathrm{H}_{\mathrm{b}}\right), 0.88\left(\mathrm{t}, J=7.1 \mathrm{~Hz}, 6 \mathrm{H}, \mathrm{C}_{10}-\mathrm{H}, \mathrm{C}_{8}-\mathrm{H}\right.$ or $\left.\mathrm{C}_{9}-\mathrm{H}\right), 0.83\left(\mathrm{dd}, J=12.3,3.4 \mathrm{~Hz}, 1 \mathrm{H}, \mathrm{C}_{4}-\mathrm{H}_{\mathrm{b}}\right), 0.73\left(\mathrm{~d}, \mathrm{~J}=6.9 \mathrm{~Hz}, 3 \mathrm{H}, \mathrm{C}_{8}-\mathrm{H}\right.$ or $\left.\mathrm{C}_{9}-\mathrm{H}\right)$; ${ }^{13} \mathrm{C}$ NMR $\left(125 \mathrm{MHz}, \mathrm{CDCl}_{3}\right) \delta 167.99,159.72(\mathrm{~d}, J=249.9 \mathrm{~Hz}), 152.21,150.82,132.67(\mathrm{~d}$, $J=8.2 \mathrm{~Hz}), 132.27,124.97(\mathrm{~d}, J=3.6 \mathrm{~Hz}), 116.07(\mathrm{~d}, J=21.3 \mathrm{~Hz}), 115.18(\mathrm{~d}, J=14.6 \mathrm{~Hz})$, 76.40, 46.92, 40.61, 35.84, 34.12, 31.41, 31.32 (d, $J=6.2 \mathrm{~Hz}), 26.19,23.37,21.96,20.73,16.26$; ESI-MS $m / z$ : $406.20[\mathrm{M}+\mathrm{H}]^{+}$. Anal. Calcd. for $\mathrm{C}_{21} \mathrm{H}_{28} \mathrm{FN}_{3} \mathrm{O}_{2} \mathrm{~S}: \mathrm{C}, 62.20 ; \mathrm{H}, 6.96 ; \mathrm{N}, 10.36$; Found: C, 61.18; H, 6.94; N, 10.32 .

Compound (5g): (1R,2S,5R)-2-isopropyl-5-methylcyclohexyl-2-((5-(3-fluorophenyl)-4-methyl4H-1,2,4-triazol-3-yl)thio) acetate. Pale yellow liquid. Yield: $65.3 \%$, IR $\left(\mathrm{KBr}_{\mathrm{cm}}{ }^{-1}\right): 2959,2933$, 2868 (s, C-H), 1736(s, C=O), 1484 (s, C=N), 683 (w, C-S-C); ${ }^{1} \mathrm{H}$ NMR $\left(500 \mathrm{MHz}, \mathrm{CDCl}_{3}\right) \delta$ $7.46\left(\mathrm{td}, J=7.9,5.6 \mathrm{~Hz}, 1 \mathrm{H}, \mathrm{C}_{19}-\mathrm{H}\right), 7.42-7.38\left(\mathrm{~m}, 1 \mathrm{H}, \mathrm{C}_{20}-\mathrm{H}\right), 7.35(\mathrm{dt}, J=9.3,2.1 \mathrm{~Hz}, 1 \mathrm{H}$, $\left.\mathrm{C}_{16}-\mathrm{H}\right), 7.17\left(\mathrm{td}, J=8.9,8.4,2.5 \mathrm{~Hz}, 1 \mathrm{H}, \mathrm{C}_{18}-\mathrm{H}\right), 4.69\left(\mathrm{td}, J=10.9,4.4 \mathrm{~Hz}, 1 \mathrm{H}, \mathrm{C}_{1}-\mathrm{H}\right), 4.06$ (s, $\left.2 \mathrm{H}, \mathrm{C}_{12}-\mathrm{H}\right), 3.66\left(\mathrm{~s}, 3 \mathrm{H}, \mathrm{C}_{21}-\mathrm{H}\right), 1.95\left(\mathrm{dtd}, J=12.1,4.0,1.7 \mathrm{~Hz}, 1 \mathrm{H}, \mathrm{C}_{6}-\mathrm{H}_{\mathrm{a}}\right), 1.80(\mathrm{pd}, J=7.0$, $\left.2.7 \mathrm{~Hz}, 1 \mathrm{H}, \mathrm{C}_{7}-\mathrm{H}\right), 1.66\left(\mathrm{q}, J=2.6,1.9 \mathrm{~Hz}, 1 \mathrm{H}, \mathrm{C}_{4}-\mathrm{H}_{\mathrm{a}}\right), 1.64-1.61\left(\mathrm{~m}, 1 \mathrm{H}, \mathrm{C}_{3}-\mathrm{H}_{\mathrm{a}}\right), 1.44$ (dddt, $\left.J=12.1,8.8,6.2,3.4 \mathrm{~Hz}, 1 \mathrm{H}, \mathrm{C}_{5}-\mathrm{H}\right), 1.36\left(\mathrm{ddt}, J=14.2,10.9,3.2 \mathrm{~Hz}, 1 \mathrm{H}, \mathrm{C}_{2}-\mathrm{H}\right), 1.06-0.98$ $\left(\mathrm{m}, 1 \mathrm{H}, \mathrm{C}_{3}-\mathrm{H}_{\mathrm{b}}\right), 0.99-0.93\left(\mathrm{~m}, 1 \mathrm{H}, \mathrm{C}_{6}-\mathrm{H}_{\mathrm{b}}\right), 0.88-0.84\left(\mathrm{~m}, 6 \mathrm{H}, \mathrm{C}_{10}-\mathrm{H}, \mathrm{C}_{8}-\mathrm{H}\right.$ or $\left.\mathrm{C}_{9}-\mathrm{H}\right), 0.83-$ $0.78\left(\mathrm{~m}, 1 \mathrm{H}, \mathrm{C}_{4}-\mathrm{H}_{\mathrm{b}}\right), 0.70\left(\mathrm{~d}, J=7.0 \mathrm{~Hz}, 3 \mathrm{H}, \mathrm{C}_{8}-\mathrm{H}\right.$ or $\left.\mathrm{C}_{9}-\mathrm{H}\right) ;{ }^{13} \mathrm{C} \mathrm{NMR}\left(125 \mathrm{MHz} \mathrm{CDCl}_{3}\right) \delta$ $168.11,162.85(\mathrm{~d}, J=248.0 \mathrm{~Hz}), 155.02,151.17,130.82(\mathrm{~d}, J=8.2 \mathrm{~Hz}), 129.03(\mathrm{~d}, J=8.5 \mathrm{~Hz})$, $124.35(\mathrm{~d}, J=3.2 \mathrm{~Hz}), 117.36(\mathrm{~d}, J=21.0 \mathrm{~Hz}), 115.86(\mathrm{~d}, J=23.3 \mathrm{~Hz}), 76.55,47.05,40.76,36.08$, 34.23, 32.06, 31.53, 26.31, 23.48, 22.08, 20.86, 16.40; ESI-MS m/z: $406.20[\mathrm{M}+\mathrm{H}]^{+}$. Anal. Calcd. for $\mathrm{C}_{21} \mathrm{H}_{28} \mathrm{FN}_{3} \mathrm{O}_{2} \mathrm{~S}$ : C, 62.20; H, 6.96; N,10.36; Found: C, 61.17; H, 6.93; N, 10.31 .

Compound (5h): (1R,2S,5R)-2-isopropyl-5-methylcyclohexyl-2-((5-(4-fluorophenyl)-4-methyl4H-1,2,4-triazol-3-yl)thio) acetate. White solid. Yield: $60.1 \%$, m.p. $136.5-137.9{ }^{\circ} \mathrm{C}$; IR (KBr, $\mathrm{cm}^{-1}$ ): 2958, 2929, 2869 (s, C-H), 1732 (s, C=O), 1484 (s, C=N), 689 (w, C-S-C). ${ }^{1} \mathrm{H}$ NMR $\left(500 \mathrm{MHz} \mathrm{CDCl}_{3}\right) \delta 7.62\left(\mathrm{dd}, J=8.8,5.2 \mathrm{~Hz}, 2 \mathrm{H}, \mathrm{C}_{20}-\mathrm{H}, \mathrm{C}_{16}-\mathrm{H}\right), 7.20(\mathrm{t}, J=8.6 \mathrm{~Hz}, 2 \mathrm{H}$, $\left.\mathrm{C}_{19}-\mathrm{H}, \mathrm{C}_{17}-\mathrm{H}\right), 4.71\left(\mathrm{td}, J=10.9,4.4 \mathrm{~Hz}, 1 \mathrm{H}, \mathrm{C}_{1}-\mathrm{H}\right), 4.07\left(\mathrm{~s}, 2 \mathrm{H}, \mathrm{C}_{12}-\mathrm{H}\right), 3.65\left(\mathrm{~s}, 3 \mathrm{H}, \mathrm{C}_{21}-\mathrm{H}\right)$, $1.98\left(\mathrm{dtd}, J=12.0,4.0,1.7 \mathrm{~Hz}, 1 \mathrm{H}, \mathrm{C}_{6}-\mathrm{H}_{\mathrm{a}}\right), 1.83\left(\mathrm{qd}, J=7.0,2.8 \mathrm{~Hz}, 1 \mathrm{H}, \mathrm{C}_{7}-\mathrm{H}\right), 1.68(\mathrm{q}$, $\left.J=2.6,1.9 \mathrm{~Hz}, 1 \mathrm{H}, \mathrm{C}_{4}-\mathrm{H}_{\mathrm{a}}\right), 1.65\left(\mathrm{q}, J=2.8 \mathrm{~Hz}, 1 \mathrm{H}, \mathrm{C}_{3}-\mathrm{H}_{\mathrm{a}}\right), 1.46(\mathrm{dddd}, J=12.0,8.9,6.4$, $\left.3.3 \mathrm{~Hz}, 1 \mathrm{H}, \mathrm{C}_{5}-\mathrm{H}\right), 1.38$ (ddt, $\left.J=14.2,10.8,3.1 \mathrm{~Hz}, 1 \mathrm{H}, \mathrm{C}_{2}-\mathrm{H}\right), 1.07-1.00\left(\mathrm{~m}, 1 \mathrm{H}, \mathrm{C}_{3}-\mathrm{H}_{\mathrm{b}}\right)$, 0.99-0.95 (m, 1H, C $\left.6-\mathrm{H}_{\mathrm{b}}\right), 0.90-0.86\left(\mathrm{~m}, 6 \mathrm{H}, \mathrm{C}_{10}-\mathrm{H}, \mathrm{C}_{8}-\mathrm{H}\right.$ or $\left.\mathrm{C}_{9}-\mathrm{H}\right), 0.86-0.81\left(\mathrm{~m}, 1 \mathrm{H}, \mathrm{C}_{4}\right.$ $\left.\mathrm{H}_{\mathrm{b}}\right), 0.72\left(\mathrm{~d}, \mathrm{~J}=6.9 \mathrm{~Hz}, 3 \mathrm{H}, \mathrm{C}_{8}-\mathrm{H}\right.$ or $\left.\mathrm{C}_{9}-\mathrm{H}\right) ;{ }^{13} \mathrm{C} \mathrm{NMR}\left(125 \mathrm{MHz}, \mathrm{CDCl}_{3}\right) \delta 168.17,163.92$ $(\mathrm{d}, J=251.1 \mathrm{~Hz}), 155.35,150.81,130.80(\mathrm{~d}, J=8.6 \mathrm{~Hz}), 123.27(\mathrm{~d}, J=3.6 \mathrm{~Hz}), 116.32$ (d, $J=22.1 \mathrm{~Hz}), 76.53,47.05,40.76,36.12,34.23,31.97,31.54,26.31,23.49$, 22.09, 20.87, 16.41; 
ESI-MS m/z: $406.19[\mathrm{M}+\mathrm{H}]^{+}$. Anal. Calcd. for $\mathrm{C}_{21} \mathrm{H}_{28} \mathrm{FN}_{3} \mathrm{O}_{2} \mathrm{~S}: \mathrm{C}, 62.20 ; \mathrm{H}, 6.96 ; \mathrm{N}, 10.36$; Found: C, 61.17; $\mathrm{H}, 6.92 ; \mathrm{N}, 10.30$.

Compound (5i): (1R,2S,5R)-2-isopropyl-5-methylcyclohexyl-2-((5-(2-chlorophenyl)-4-methyl4H-1,2,4-triazol-3-yl)thio)acetate. Pale yellow liquid. Yield: $60.8 \%$; IR $\left(\mathrm{KBr}, \mathrm{cm}^{-1}\right): 2954,2927$, 2869 (s, C-H), 1731 (s, C=O), 1467 (s, C=N), 698 (w, C-S-C). ${ }^{1} \mathrm{H}$ NMR (500 MHz, CDCl 3 ) $\delta$ 7.54-7.44 (m, 3H, Ar-H), 7.42- 7.36 (m, 1H, Ar-H), $4.73\left(\mathrm{td}, J=10.9,4.4 \mathrm{~Hz}, 1 \mathrm{H}, \mathrm{C}_{1}-\mathrm{H}\right), 4.11$ $\left(\mathrm{s}, 2 \mathrm{H}, \mathrm{C}_{12}-\mathrm{H}\right), 3.48\left(\mathrm{~s}, 3 \mathrm{H}, \mathrm{C}_{21}-\mathrm{H}\right), 1.98\left(\mathrm{dd}, J=10.7,5.9 \mathrm{~Hz}, 1 \mathrm{H}, \mathrm{C}_{6}-\mathrm{H}_{\mathrm{a}}\right), 1.87(\mathrm{dt}, J=7.1$, $\left.3.5 \mathrm{~Hz}, 1 \mathrm{H}, \mathrm{C}_{7}-\mathrm{H}\right), 1.68\left(\mathrm{q}, J=2.8,2.4 \mathrm{~Hz}, 1 \mathrm{H}, \mathrm{C}_{4}-\mathrm{H}_{\mathrm{a}}\right), 1.65\left(\mathrm{q}, J=2.8 \mathrm{~Hz}, 1 \mathrm{H}, \mathrm{C}_{3}-\mathrm{H}_{\mathrm{a}}\right), 1.46$ $\left(\mathrm{ddt}, J=8.9,5.7,2.8 \mathrm{~Hz}, 1 \mathrm{H}, \mathrm{C}_{5}-\mathrm{H}\right), 1.42-1.35\left(\mathrm{~m}, 1 \mathrm{H}, \mathrm{C}_{2}-\mathrm{H}\right), 1.05(\mathrm{ddd}, J=13.1,9.7,3.1 \mathrm{~Hz}$, $\left.1 \mathrm{H}, \mathrm{C}_{3}-\mathrm{H}_{\mathrm{b}}\right), 1.01-0.95\left(\mathrm{~m}, 1 \mathrm{H}, \mathrm{C}_{6}-\mathrm{H}_{\mathrm{b}}\right), 0.91-0.87\left(\mathrm{~m}, 6 \mathrm{H}, \mathrm{C}_{10}-\mathrm{H}, \mathrm{C}_{8}-\mathrm{H}\right.$ or $\left.\mathrm{C}_{9}-\mathrm{H}\right), 0.87-0.82(\mathrm{~m}$, $\left.1 \mathrm{H}, \mathrm{C}_{4}-\mathrm{H}_{\mathrm{b}}\right), 0.74\left(\mathrm{~d}, J=7.0 \mathrm{~Hz}, 3 \mathrm{H}, \mathrm{C}_{8}-\mathrm{H}\right.$ or $\left.\mathrm{C}_{9}-\mathrm{H}\right) ;{ }^{13} \mathrm{C}$ NMR $(125 \mathrm{MHz}, \mathrm{CDCl} 3) \delta 167.99$, $154.22,150.37,134.23,132.65,131.97,129.89$, 127.26, 126.58, 76.39, 46.93, 40.63, 35.85, 34.12, 31.41, 31.11, 26.20, 23.37, 21.98, 20.75, 16.29; ESI-MS m/z: C $422.17[\mathrm{M}+\mathrm{H}]^{+}$. Anal. Calcd. for $\mathrm{C}_{21} \mathrm{H}_{28} \mathrm{ClN}_{3} \mathrm{O}_{2} \mathrm{~S}$ : C, 59.77; $\mathrm{H}, 6.69 ; \mathrm{N}, 9.96$; Found: C, 59.76; $\mathrm{H}, 6.67 ; \mathrm{N}, 9.93$.

Compound (5j): (1R,2S,5R)-2-isopropyl-5-methylcyclohexyl-2-((5-(3-chlorophenyl)-4-methyl4H-1,2,4-triazol-3-yl)thio)acetate. White solid. Yield: $72.1 \%$, m.p. $68.4-70.0^{\circ} \mathrm{C} ; \mathrm{IR}\left(\mathrm{KBr}, \mathrm{cm}^{-1}\right)$ : 2955, 2935, 2869 (s, C-H), 1732 (s, C=O), 1471 (s, C=N), 685 (s, C-S-C). ${ }^{1} \mathrm{H}$ NMR (500 MHz, $\left.\mathrm{CDCl}_{3}\right) \delta 7.64\left(\mathrm{~d}, J=2.0 \mathrm{~Hz}, 1 \mathrm{H}, \mathrm{C}_{20}-\mathrm{H}\right), 7.52\left(\mathrm{dt}, J=7.4,1.7 \mathrm{~Hz}, 1 \mathrm{H}, \mathrm{C}_{16}-\mathrm{H}\right), 7.48(\mathrm{dt}$, $\left.J=8.1,1.7 \mathrm{~Hz}, 1 \mathrm{H}, \mathrm{C}_{19}-\mathrm{H}\right), 7.46-7.42\left(\mathrm{~m}, 1 \mathrm{H}, \mathrm{C}_{18}-\mathrm{H}\right), 4.71\left(\mathrm{td}, J=10.9,4.4 \mathrm{~Hz}, 1 \mathrm{H}, \mathrm{C}_{1}-\mathrm{H}\right)$, $4.08\left(\mathrm{~s}, 2 \mathrm{H}, \mathrm{C}_{12}-\mathrm{H}\right), 3.67\left(\mathrm{~s}, 3 \mathrm{H}, \mathrm{C}_{21}-\mathrm{H}\right), 1.97\left(\mathrm{dtd}, J=12.1,4.1,1.7 \mathrm{~Hz}, 1 \mathrm{H}, \mathrm{C}_{6}-\mathrm{H}_{\mathrm{a}}\right), 1.82(\mathrm{qt}$, $\left.J=7.0,3.5 \mathrm{~Hz}, 1 \mathrm{H}, \mathrm{C}_{7}-\mathrm{H}\right), 1.68\left(\mathrm{q}, J=2.6,1.9 \mathrm{~Hz}, 1 \mathrm{H}, \mathrm{C}_{4}-\mathrm{H}_{\mathrm{a}}\right), 1.65\left(\mathrm{q}, J=2.9 \mathrm{~Hz}, 1 \mathrm{H}, \mathrm{C}_{3}-\mathrm{H}_{\mathrm{a}}\right)$, 1.46 (dddt, $\left.J=12.1,8.9,6.1,3.3 \mathrm{~Hz}, 1 \mathrm{H}, \mathrm{C}_{5}-\mathrm{H}\right), 1.38\left(\mathrm{ddt}, J=14.2,10.9,3.1 \mathrm{~Hz}, 1 \mathrm{H}, \mathrm{C}_{2}-\mathrm{H}\right)$, 1.10-1.01 (m, 1H, $\left.\mathrm{C}_{3}-\mathrm{H}_{\mathrm{b}}\right), 1.01-0.95\left(\mathrm{~m}, 1 \mathrm{H}, \mathrm{C}_{6}-\mathrm{H}_{\mathrm{b}}\right), 0.88\left(\mathrm{t}, J=7.2 \mathrm{~Hz}, 6 \mathrm{H}, \mathrm{C}_{10}-\mathrm{H}, \mathrm{C}_{8}-\mathrm{H}\right.$ or $\left.\mathrm{C}_{9}-\mathrm{H}\right), 0.86-0.81\left(\mathrm{~m}, 1 \mathrm{H}, \mathrm{C}_{4}-\mathrm{H}_{\mathrm{b}}\right), 0.73\left(\mathrm{~d}, J=7.0 \mathrm{~Hz}, 3 \mathrm{H}, \mathrm{C}_{8}-\mathrm{H}\right.$ or $\left.\mathrm{C}_{9}-\mathrm{H}\right) ;{ }^{13} \mathrm{C}$ NMR $(125 \mathrm{MHz}$, $\left.\mathrm{CDCl}_{3}\right) \delta 167.98,154.79,151.06,134.99,130.30,130.25,128.66,128.63,126.65,76.43,46.93$, $40.64,35.98,34.11,31.93,31.41,26.19,23.36,21.97,20.75,16.29$; ESI-MS $m / z: 422.18[\mathrm{M}+\mathrm{H}]^{+}$. Anal. Calcd. for $\mathrm{C}_{21} \mathrm{H}_{28} \mathrm{ClN}_{3} \mathrm{O}_{2} \mathrm{~S}: \mathrm{C}, 59.77 ; \mathrm{H}, 6.69 ; \mathrm{N}, 9.96$; Found: $\mathrm{C}, 59.75 ; \mathrm{H}, 6.65 ; \mathrm{N}, 9.92$.

Compound (5k): (1R,2S,5R)-2-isopropyl-5-methylcyclohexyl-2-((5-(2-chlorophenyl)-4-methyl4H-1,2,4-triazol-3-yl)thio)acetate. White solid. Yield: $66.2 \%$, m.p. $121.9-123.7^{\circ} \mathrm{C}$; IR (KBr, $\mathrm{v} / \mathrm{cm}^{-1}$ ): 2956, 2932, 2869 (s, C-H), 1732(s, C=O), 1478 (s, C=N), 713 (w, C-S-C). ${ }^{1} \mathrm{H}$ NMR $\left(500 \mathrm{MHz}, \mathrm{CDCl}_{3}\right) \delta 7.58\left(\mathrm{~d}, J=8.5 \mathrm{~Hz}, 2 \mathrm{H}, \mathrm{C}_{16}-\mathrm{H}, \mathrm{C}_{20}-\mathrm{H}\right), 7.48\left(\mathrm{~d}, J=8.6 \mathrm{~Hz}, 2 \mathrm{H}, \mathrm{C}_{17}-\mathrm{H}\right.$, $\left.\mathrm{C}_{19}-\mathrm{H}\right), 4.71\left(\mathrm{td}, J=10.9,4.4 \mathrm{~Hz}, 1 \mathrm{H}, \mathrm{C}_{1}-\mathrm{H}\right), 4.07\left(\mathrm{~s}, 2 \mathrm{H}, \mathrm{C}_{12}-\mathrm{H}\right), 3.66\left(\mathrm{~s}, 3 \mathrm{H}, \mathrm{C}_{21}-\mathrm{H}\right), 1.97$ $\left(\mathrm{dtd}, J=12.1,4.1,1.8 \mathrm{~Hz}, 1 \mathrm{H}, \mathrm{C}_{6}-\mathrm{H}_{\mathrm{a}}\right), 1.81\left(\mathrm{qd}, J=7.0,2.7 \mathrm{~Hz}, 1 \mathrm{H}, \mathrm{C}_{7}-\mathrm{H}\right), 1.68(\mathrm{q}, J=2.6$, $\left.1.9 \mathrm{~Hz}, 1 \mathrm{H}, \mathrm{C}_{4}-\mathrm{H}_{\mathrm{a}}\right), 1.65\left(\mathrm{q}, J=2.9 \mathrm{~Hz}, 1 \mathrm{H}, \mathrm{C}_{3}-\mathrm{H}_{\mathrm{a}}\right), 1.46(\mathrm{dddt}, J=12.0,8.9,6.2,3.4 \mathrm{~Hz}, 1 \mathrm{H}$, $\left.\mathrm{C}_{5}-\mathrm{H}\right), 1.41-1.35\left(\mathrm{~m}, 1 \mathrm{H}, \mathrm{C}_{2}-\mathrm{H}\right), 1.09-1.01\left(\mathrm{~m}, 1 \mathrm{H}, \mathrm{C}_{3}-\mathrm{H}_{\mathrm{b}}\right), 1.00-0.95\left(\mathrm{~m}, 1 \mathrm{H}, \mathrm{C}_{6}-\mathrm{H}_{\mathrm{b}}\right), 0.88$ $\left(\mathrm{dd}, J=8.5,6.7 \mathrm{~Hz}, 6 \mathrm{H}, \mathrm{C}_{10}-\mathrm{H}, \mathrm{C}_{8}-\mathrm{H}\right.$ or $\left.\mathrm{C}_{9}-\mathrm{H}\right), 0.86-0.79\left(\mathrm{~m}, 1 \mathrm{H}, \mathrm{C}_{4}-\mathrm{H}_{\mathrm{b}}\right), 0.72(\mathrm{~d}, J=7.0 \mathrm{~Hz}$, $3 \mathrm{H}, \mathrm{C}_{8}-\mathrm{H}$ or $\left.\mathrm{C}_{9}-\mathrm{H}\right) ;{ }^{13} \mathrm{C}$ NMR $\left(125 \mathrm{MHz}, \mathrm{CDCl}_{3}\right) \delta 168.01,155.09,150.92,136.47,129.83$, $129.28,125.43,76.42,46.93,40.63,35.98,34.10,31.90,31.41,26.18,23.36,21.97,20.74,16.28$; ESI-MS $m / z: 422.17[\mathrm{M}+\mathrm{H}]^{+}$. Anal. Calcd. for $\mathrm{C}_{21} \mathrm{H}_{28} \mathrm{ClN}_{3} \mathrm{O}_{2} \mathrm{~S}: \mathrm{C}, 59.77 ; \mathrm{H}, 6.69 ; \mathrm{N}, 9.96$; Found: C, 59.74; H, 6.63; N, 9.93 .

Compound (5l): (1R,2S,5R)-2-isopropyl-5-methylcyclohexyl-2-((5-(4-bromophenyl)-4-methyl4H-1,2,4-triazol-3-yl)thio)acetate. White solid. Yield: 67.1\%, m.p. $144.4-145.2{ }^{\circ} \mathrm{C}$; IR (KBr, $\mathrm{cm}^{-1}$ ): 2955, 2931, 2870 (s, C-H), 1731 (s, C=O), 1477 (s, C=N), 713 (w, C-S-C). ${ }^{1} \mathrm{H}$ NMR (500 MHz, $\left.\mathrm{CDCl}_{3}\right) \delta$ 7.68-7.62 (m, 2H, $\left.\mathrm{C}_{16}-\mathrm{H}, \mathrm{C}_{20}-\mathrm{H}\right), 7.54-7.48\left(\mathrm{~m}, 2 \mathrm{H}, \mathrm{C}_{17}-\mathrm{H}, \mathrm{C}_{19}-\mathrm{H}\right), 4.71$ $\left(\mathrm{td}, J=10.9,4.4 \mathrm{~Hz}, 1 \mathrm{H}, \mathrm{C}_{1}-\mathrm{H}\right), 4.08\left(\mathrm{~s}, 2 \mathrm{H}, \mathrm{C}_{12}-\mathrm{H}\right), 3.66\left(\mathrm{~s}, 3 \mathrm{H}, \mathrm{C}_{21}-\mathrm{H}\right), 1.97(\mathrm{dtd}, J=12.1$, $\left.4.2,1.8 \mathrm{~Hz}, 1 \mathrm{H}, \mathrm{C}_{6}-\mathrm{Ha}\right), 1.82\left(\mathrm{pd}, J=7.0,2.8 \mathrm{~Hz}, 1 \mathrm{H}, \mathrm{C}_{7}-\mathrm{H}\right), 1.68(\mathrm{q}, J=2.6,1.9 \mathrm{~Hz}, 1 \mathrm{H}$, $\left.\mathrm{C}_{4}-\mathrm{Ha}\right), 1.65\left(\mathrm{q}, J=2.9 \mathrm{~Hz}, 1 \mathrm{H}, \mathrm{C}_{3}-\mathrm{Ha}\right), 1.46$ (dddd, $\left.J=12.1,8.9,6.2,3.2 \mathrm{~Hz}, 1 \mathrm{H}, \mathrm{C}_{5}-\mathrm{H}\right)$, $1.38\left(\mathrm{ddt}, J=14.2,10.9,3.1 \mathrm{~Hz}, 1 \mathrm{H}, \mathrm{C}_{2}-\mathrm{H}\right), 1.08-1.01\left(\mathrm{~m}, 1 \mathrm{H}, \mathrm{C}_{3}-\mathrm{H}_{\mathrm{b}}\right), 1.00-0.95(\mathrm{~m}, 1 \mathrm{H}$, $\left.\mathrm{C}_{6}-\mathrm{H}_{\mathrm{b}}\right), 0.88\left(\mathrm{dd}, J=8.7,6.7 \mathrm{~Hz}, 6 \mathrm{H}, \mathrm{C}_{10}-\mathrm{H}, \mathrm{C}_{8}-\mathrm{H}\right.$ or $\left.\mathrm{C}_{9}-\mathrm{H}\right), 0.86-0.81\left(\mathrm{~m}, 1 \mathrm{H}, \mathrm{C}_{4}-\mathrm{H}_{\mathrm{b}}\right), 0.72$ $\left(\mathrm{d}, J=6.9 \mathrm{~Hz}, 3 \mathrm{H}, \mathrm{C}_{8}-\mathrm{H}\right.$ or $\left.\mathrm{C}_{9}-\mathrm{H}\right) ;{ }^{13} \mathrm{C}$ NMR $\left(125 \mathrm{MHz}, \mathrm{CDCl}_{3}\right) \delta 168.00,155.13,150.97$, $132.24,130.02,125.88,124.74,76.42,46.92,40.63,35.97,34.10,31.91,31.41,26.18,23.35,21.97$, 20.74, 16.28; ESI-MS $m / z: 466.13[\mathrm{M}+\mathrm{H}]^{+}$. Anal. Calcd. for $\mathrm{C}_{21} \mathrm{H}_{28} \mathrm{BrN}_{3} \mathrm{O}_{2} \mathrm{~S}: \mathrm{C}, 54.08 ; \mathrm{H}$, 6.05; N,9.01; Found: C, 54.06; H, 6.02; N, 9.00. 
Compound (5m): (1R,2S,5R)-2-isopropyl-5-methylcyclohexyl-2-((5-(2-iodophenyl)-4-methyl4H-1,2,4-triazol-3-yl)thio)acetate. Pale yellow liquid. Yield: 50.6\%; IR (KBr, cm $\left.{ }^{-1}\right): 2954,2927$, 2868 (s, C-H), 1731 (s, C=O), 1466 (s, C=N), 692 (w, C-S-C); ${ }^{1} \mathrm{H}$ NMR (500 MHz, CDCl $\left.{ }_{3}\right) \delta$ $7.95\left(\mathrm{~d}, J=8.0 \mathrm{~Hz}, 1 \mathrm{H}, \mathrm{C}_{17}-\mathrm{H}\right), 7.50-7.46\left(\mathrm{~m}, 1 \mathrm{H}, \mathrm{C}_{18}-\mathrm{H}\right), 7.38\left(\mathrm{dd}, J=7.6,1.7 \mathrm{~Hz}, 1 \mathrm{H}, \mathrm{C}_{20}-\mathrm{H}\right)$, $7.22\left(\mathrm{td}, J=7.7,1.8 \mathrm{~Hz}, 1 \mathrm{H}, \mathrm{C}_{19}-\mathrm{H}\right), 4.73\left(\mathrm{td}, J=11.0,4.4 \mathrm{~Hz}, 1 \mathrm{H}, \mathrm{C}_{1}-\mathrm{H}\right), 4.12\left(\mathrm{~s}, 2 \mathrm{H}, \mathrm{C}_{12}-\mathrm{H}\right)$, $3.44\left(\mathrm{~s}, 3 \mathrm{H}, \mathrm{C}_{21}-\mathrm{H}\right), 2.00\left(\mathrm{dtd}, J=12.0,4.0,1.8 \mathrm{~Hz}, 1 \mathrm{H}, \mathrm{C}_{6}-\mathrm{H}_{\mathrm{a}}\right), 1.88(\mathrm{pd}, J=7.0,2.7 \mathrm{~Hz}, 1 \mathrm{H}$, $\left.\mathrm{C}_{7}-\mathrm{H}\right), 1.69\left(\mathrm{q}, J=2.8 \mathrm{~Hz}, 1 \mathrm{H}, \mathrm{C}_{4}-\mathrm{H}_{\mathrm{a}}\right), 1.66\left(\mathrm{~h}, J=2.8 \mathrm{~Hz}, 1 \mathrm{H}, \mathrm{C}_{3}-\mathrm{H}_{\mathrm{a}}\right), 1.47$ (dddt, $J=15.3,8.8$, 6.2, 3.2 Hz, 1H, $\left.\mathrm{C}_{5}-\mathrm{H}\right), 1.43-1.37\left(\mathrm{~m}, 1 \mathrm{H}, \mathrm{C}_{2}-\mathrm{H}\right), 1.10-1.03\left(\mathrm{~m}, 1 \mathrm{H}, \mathrm{C}_{3}-\mathrm{H}_{\mathrm{b}}\right), 1.03-0.97(\mathrm{~m}, 1 \mathrm{H}$, $\left.\mathrm{C}_{6}-\mathrm{H}_{\mathrm{b}}\right), 0.90\left(\mathrm{~d}, \mathrm{~J}=3.4 \mathrm{~Hz}, 3 \mathrm{H}, \mathrm{C}_{10}-\mathrm{H}\right), 0.89\left(\mathrm{~d}, \mathrm{~J}=3.9 \mathrm{~Hz}, 3 \mathrm{H}, \mathrm{C}_{8}-\mathrm{H}\right.$ or $\left.\mathrm{C}_{9}-\mathrm{H}\right), 0.87-0.84(\mathrm{~m}$, $\left.1 \mathrm{H}, \mathrm{C}_{4}-\mathrm{H}_{\mathrm{b}}\right), 0.75\left(\mathrm{~d}, \mathrm{~J}=7.0 \mathrm{~Hz}, 3 \mathrm{H}, \mathrm{C}_{8}-\mathrm{H}\right.$ or $\left.\mathrm{C}_{9}-\mathrm{H}\right) ;{ }^{13} \mathrm{C} \mathrm{NMR}\left(125 \mathrm{MHz}, \mathrm{CDCl}_{3}\right) \delta 168.11$, 157.37, 150.15, 139.51, 133.05, 132.13, 132.08, 128.53, 98.86, 76.52, 47.06, 40.79, 36.04, 34.25, 31.54, 31.39, 26.32, 23.50, 22.12, 20.90, 16.45; ESI-MS m/z: $514.13[\mathrm{M}+\mathrm{H}]^{+}$. Anal. Calcd. for $\mathrm{C}_{21} \mathrm{H}_{28} \mathrm{IN}_{3} \mathrm{O}_{2} \mathrm{~S}: \mathrm{C}, 49.13 ; \mathrm{H}, 5.50 ; \mathrm{N}, 8.18$; Found: $\mathrm{C}, 49.11 ; \mathrm{H}, 5.48 ; \mathrm{N}, 8.15$.

Compound (5n): (1R,2S,5R)-2-isopropyl-5-methylcyclohexyl-2-((5-(4-iodophenyl)-4-methyl4H-1,2,4-triazol-3-yl)thio) acetate. White solid. Yield: $67.1 \%$, m.p. $131.1-131.8{ }^{\circ} \mathrm{C}$; IR (KBr, $\mathrm{cm}^{-1}$ ): 2954, 2929, 2869 (s, C-H), 1731 (s, C=O), 1475 (s, C=N), 710 (w, C-S-C); ${ }^{1} \mathrm{H}$ NMR $\left(500 \mathrm{MHz}, \mathrm{CDCl}_{3}\right) \delta 7.89-7.81\left(\mathrm{~m}, 2 \mathrm{H}, \mathrm{C}_{17}-\mathrm{H}, \mathrm{C}_{19}-\mathrm{H}\right), 7.41-7.34\left(\mathrm{~m}, 2 \mathrm{H}, \mathrm{C}_{16}-\mathrm{H}, \mathrm{C}_{20}-\mathrm{H}\right), 4.70$ $\left(\mathrm{td}, J=10.9,4.4 \mathrm{~Hz}, 1 \mathrm{H}, \mathrm{C}_{1}-\mathrm{H}\right), 4.07\left(\mathrm{~s}, 2 \mathrm{H}, \mathrm{C}_{12}-\mathrm{H}\right), 3.65\left(\mathrm{~s}, 3 \mathrm{H}, \mathrm{C}_{21}-\mathrm{H}\right), 1.97$ (dtd, J = 12.0, 4.0, $\left.1.7 \mathrm{~Hz}, 1 \mathrm{H}, \mathrm{C}_{6}-\mathrm{H}_{\mathrm{a}}\right), 1.82\left(\mathrm{pd}, J=7.1,2.9 \mathrm{~Hz}, 1 \mathrm{H}, \mathrm{C}_{7}-\mathrm{H}\right), 1.68(\mathrm{q}, J=2.5,1.9 \mathrm{~Hz}, 1 \mathrm{H}$, $\left.\mathrm{C}_{4}-\mathrm{H}_{\mathrm{a}}\right), 1.66-1.63\left(\mathrm{~m}, 1 \mathrm{H}, \mathrm{C}_{3}-\mathrm{H}_{\mathrm{a}}\right), 1.45$ (dddt, $\left.J=12.2,9.5,6.5,3.2 \mathrm{~Hz}, 1 \mathrm{H}, \mathrm{C}_{5}-\mathrm{H}\right), 1.38$ (ddt, $\left.J=14.1,10.9,3.2 \mathrm{~Hz}, 1 \mathrm{H}, \mathrm{C}_{2}-\mathrm{H}\right), 1.08-1.00\left(\mathrm{~m}, 1 \mathrm{H}, \mathrm{C}_{3}-\mathrm{H}_{\mathrm{b}}\right), 1.00-0.95\left(\mathrm{~m}, 1 \mathrm{H}, \mathrm{C}_{6}-\mathrm{H}_{\mathrm{b}}\right), 0.89$ $\left(\mathrm{d}, J=6.5 \mathrm{~Hz}, 3 \mathrm{H}, \mathrm{C}_{10}-\mathrm{H}\right), 0.87\left(\mathrm{~d}, J=7.0 \mathrm{~Hz}, 3 \mathrm{H}, \mathrm{C}_{8}-\mathrm{H}\right.$ or $\left.\mathrm{C}_{9}-\mathrm{H}\right), 0.86-0.81\left(\mathrm{~m}, 1 \mathrm{H}, \mathrm{C}_{4}-\mathrm{H}_{\mathrm{b}}\right)$, $0.72\left(\mathrm{~d}, J=7.0 \mathrm{~Hz}, 3 \mathrm{H}, \mathrm{C}_{8}-\mathrm{H}\right.$ or $\left.\mathrm{C}_{9}-\mathrm{H}\right) ;{ }^{13} \mathrm{C} \mathrm{NMR}\left(125 \mathrm{MHz}, \mathrm{CDCl}_{3}\right) \delta 168.00,155.24,151.00$, $138.17,130.04,126.42,96.62,76.43,46.93,40.64,35.97,34.10,31.91,31.41,26.18,23.36,21.97$, 20.75, 16.28. ESI-MS $m / z: 514.11[\mathrm{M}+\mathrm{H}]^{+}$. Anal. Calcd. for $\mathrm{C}_{21} \mathrm{H}_{28} \mathrm{IN}_{3} \mathrm{O}_{2} \mathrm{~S}: \mathrm{C}, 49.13 ; \mathrm{H}$, 5.50; N, 8.18; Found: C, 49.10; H, 5.49; N, 8.16.

Compound (5o): (1R,2S,5R)-2-isopropyl-5-methylcyclohexyl-2-((4-methyl-5-(2-(trifluoromethyl) phenyl)-4H-1,2,4-triazol-3-yl)thio)acetate. White solid. Yield: 56.1\%, m.p. 88.6-90.2 ${ }^{\circ} \mathrm{C}$; IR (KBr, $\mathrm{cm}^{-1}$ ): 2954, 2929, 2869 (s, C-H), 1731 (s, C=O), 1475 (s, C=N), 710 (w, C-S-C); ${ }^{1} \mathrm{H}$ NMR $\left(500 \mathrm{MHz} \mathrm{CDCl}_{3}\right) \delta 7.88-7.80\left(\mathrm{~m}, 1 \mathrm{H}, \mathrm{C}_{20}-\mathrm{H}\right), 7.72-7.64\left(\mathrm{~m}, 2 \mathrm{H}, \mathrm{C}_{17}-\mathrm{H}, \mathrm{C}_{19}-\mathrm{H}\right), 7.49-7.43$ $\left(\mathrm{m}, 1 \mathrm{H}, \mathrm{C}_{18}-\mathrm{H}\right), 4.73\left(\mathrm{td}, \mathrm{J}=10.9,4.4 \mathrm{~Hz}, 1 \mathrm{H}, \mathrm{C}_{1}-\mathrm{H}\right), 4.11\left(\mathrm{~s}, 2 \mathrm{H}, \mathrm{C}_{12}-\mathrm{H}\right), 3.38\left(\mathrm{~s}, 3 \mathrm{H}, \mathrm{C}_{21}-\mathrm{H}\right)$, $1.98\left(\mathrm{dd}, J=10.6,5.9 \mathrm{~Hz}, 1 \mathrm{H}, \mathrm{C}_{6}-\mathrm{H}_{\mathrm{a}}\right), 1.88\left(\mathrm{ddt}, J=14.0,7.0,3.5 \mathrm{~Hz}, 1 \mathrm{H}, \mathrm{C}_{7}-\mathrm{H}\right), 1.69$ (q, $\left.J=2.9 \mathrm{~Hz}, 1 \mathrm{H}, \mathrm{C}_{4}-\mathrm{H}_{\mathrm{a}}\right), 1.66\left(\mathrm{q}, J=3.3,2.6 \mathrm{~Hz}, 1 \mathrm{H}, \mathrm{C}_{3}-\mathrm{H}_{\mathrm{a}}\right), 1.48(\mathrm{dddq}, J=12.0,8.9,6.1$, $\left.3.2 \mathrm{~Hz}, 1 \mathrm{H}, \mathrm{C}_{5}-\mathrm{H}\right), 1.40$ (ddt, $\left.J=13.8,11.0,3.1 \mathrm{~Hz}, 1 \mathrm{H}, \mathrm{C}_{2}-\mathrm{H}\right), 1.09-1.02\left(\mathrm{~m}, 1 \mathrm{H}, \mathrm{C}_{3}-\mathrm{H}_{\mathrm{b}}\right)$, 1.02-0.97 (m, 1H, $\left.\mathrm{C}_{6}-\mathrm{H}_{\mathrm{b}}\right), 0.89\left(\mathrm{dd}, J=6.8,4.9 \mathrm{~Hz}, 6 \mathrm{H}, \mathrm{C}_{10}-\mathrm{H}, \mathrm{C}_{8}-\mathrm{H}\right.$ or $\left.\mathrm{C}_{9}-\mathrm{H}\right), 0.84(\mathrm{dd}$, $\left.J=12.3,3.4 \mathrm{~Hz}, 1 \mathrm{H}, \mathrm{C}_{4}-\mathrm{H}_{\mathrm{b}}\right), 0.75\left(\mathrm{~d}, J=6.9 \mathrm{~Hz}, 3 \mathrm{H}, \mathrm{C}_{8}-\mathrm{H}\right.$ or $\left.\mathrm{C}_{9}-\mathrm{H}\right) ;{ }^{13} \mathrm{C} \mathrm{NMR}(125 \mathrm{MHz}$, $\left.\mathrm{CDCl}_{3}\right) \delta 168.11,153.42,150.34,132.54,132.16,131.06,131.02,127.21,125.59,124.57,76.56$, 47.04, 40.72, 36.06, 34.25, 31.55, 31.11, 26.3, 23.51, 22.07, 20.85, 16.39; ESI-MS m/z: 456.19 $[\mathrm{M}+\mathrm{H}]^{+}$. Anal. Calcd. for $\mathrm{C}_{22} \mathrm{H}_{28} \mathrm{~F}_{3} \mathrm{~N}_{3} \mathrm{O}_{2} \mathrm{~S}: \mathrm{C}, 58.01 ; \mathrm{H}, 6.20 ; \mathrm{N}, 9.22 ;$ Found: $\mathrm{C}, 58.00 ; \mathrm{H}$, $6.19 ; \mathrm{N}, 9.21$.

Compound (5p): (1R,2S,5R)-2-isopropyl-5-methylcyclohexyl-2-((4-methyl-5-(3-(trifluoromethyl) phenyl)-4H-1,2,4-triazol-3-yl)thio)acetate. White solid. Yield: 70.1\%, m.p. 81.5-82.3 ${ }^{\circ} \mathrm{C}$; IR (KBr, $\mathrm{cm}^{-1}$ ): 2961, 2928, 2869 (s, C-H), 1735 (s, C=O), 1459 (s, C=N), 704 (w, C-S-C); ${ }^{1} \mathrm{H}$ NMR $\left(500 \mathrm{MHz}, \mathrm{CDCl}_{3}\right) \delta 7.92\left(\mathrm{~d}, J=2.0 \mathrm{~Hz}, 1 \mathrm{H}, \mathrm{C}_{16}-\mathrm{H}\right), 7.84\left(\mathrm{~d}, J=7.7 \mathrm{~Hz}, 1 \mathrm{H}, \mathrm{C}_{20}-\mathrm{H}\right), 7.76(\mathrm{~d}$, $\left.J=7.9 \mathrm{~Hz}, 1 \mathrm{H}, \mathrm{C}_{18}-\mathrm{H}\right), 7.65\left(\mathrm{t}, J=7.8 \mathrm{~Hz}, 1 \mathrm{H}, \mathrm{C}_{19}-\mathrm{H}\right), 4.71\left(\mathrm{td}, J=10.9,4.4 \mathrm{~Hz}, 1 \mathrm{H}, \mathrm{C}_{1}-\mathrm{H}\right)$, 4.09 (s, 2H, C $12-\mathrm{H}), 3.69$ (s, 3H, C $21-\mathrm{H}), 1.98$ (dtd, J = 12.0, 4.0, 1.7 Hz, 1H, C $6-\mathrm{H}_{\mathrm{a}}$ ), 1.83 (qt, $\left.J=7.0,3.5 \mathrm{~Hz}, 1 \mathrm{H}, \mathrm{C}_{7}-\mathrm{H}\right), 1.68\left(\mathrm{q}, J=2.7,2.0 \mathrm{~Hz}, 1 \mathrm{H}, \mathrm{C}_{4}-\mathrm{H}_{\mathrm{a}}\right), 1.65\left(\mathrm{q}, J=2.8 \mathrm{~Hz}, 1 \mathrm{H}, \mathrm{C}_{3}-\mathrm{H}_{\mathrm{a}}\right)$, 1.46 (dddt, $\left.J=12.1,9.5,6.6,3.2 \mathrm{~Hz}, 1 \mathrm{H}, \mathrm{C}_{5}-\mathrm{H}\right), 1.42-1.35\left(\mathrm{~m}, 1 \mathrm{H}, \mathrm{C}_{2}-\mathrm{H}\right), 1.08-1.01(\mathrm{~m}, 1 \mathrm{H}$, $\left.\mathrm{C}_{3}-\mathrm{H}_{\mathrm{b}}\right), 1.01-0.96\left(\mathrm{~m}, 1 \mathrm{H}, \mathrm{C}_{6}-\mathrm{H}_{\mathrm{b}}\right), 0.88\left(\mathrm{t}, J=6.6 \mathrm{~Hz}, 6 \mathrm{H}, \mathrm{C}_{10}-\mathrm{H}, \mathrm{C}_{8}-\mathrm{H}\right.$ or $\left.\mathrm{C}_{9}-\mathrm{H}\right), 0.86-0.81$ $\left(\mathrm{m}, 1 \mathrm{H}, \mathrm{C}_{4}-\mathrm{H}_{\mathrm{b}}\right), 0.73\left(\mathrm{~d}, \mathrm{~J}=7.0 \mathrm{~Hz}, 3 \mathrm{H}, \mathrm{C}_{8}-\mathrm{H}\right.$ or $\left.\mathrm{C}_{9}-\mathrm{H}\right) ;{ }^{13} \mathrm{C}$ NMR $\left(125 \mathrm{MHz} \mathrm{CDCl}_{3}\right) \delta$ $168.09,154.86,151.45,132.48,129.74,128.00,127.00(q, J=3.8 \mathrm{~Hz}), 125.56(q, J=3.9 \mathrm{~Hz})$, $125.15,76.60,47.06,40.77,36.11,34.23,32.06,31.54,26.33,23.49,22.08,20.86,16.41$; ESI-MS $m / z: 456.22[\mathrm{M}+\mathrm{H}]^{+}$. Anal. Calcd. for $\mathrm{C}_{22} \mathrm{H}_{28} \mathrm{~F}_{3} \mathrm{~N}_{3} \mathrm{O}_{2} \mathrm{~S}: \mathrm{C}, 58.01 ; \mathrm{H}, 6.20 ; \mathrm{N}, 9.22 ;$ Found: $\mathrm{C}$, $57.98 ; \mathrm{H}, 6.18 ; \mathrm{N}, 9.20$. 
Compound (5q): (1R,2S,5R)-2-isopropyl-5-methylcyclohexyl-2-((5-(2-hydroxyphenyl)-4methyl-4H-1,2,4-triazol-3-yl)thio)acetate. White solid. Yield: $65.1 \%$, m.p. $158.9-160.9^{\circ} \mathrm{C}$; IR $\left(\mathrm{KBr}, \mathrm{cm}^{-1}\right.$ ): 3429(w, O-H), 2961, 2942, 2914, 2864 (s, C-H), 1735 (s, C=O), 1486 (s, C=N), 683 (w, C-S-C); ${ }^{1} \mathrm{H}$ NMR (500 MHz, CDCl 3 ) $\delta 11.18(\mathrm{~s}, 1 \mathrm{H}, \mathrm{OH}-\mathrm{H}), 7.53(\mathrm{dd}, J=8.0,1.6 \mathrm{~Hz}$, $\left.1 \mathrm{H}, \mathrm{C}_{20}-\mathrm{H}\right), 7.39-7.31\left(\mathrm{~m}, 1 \mathrm{H}, \mathrm{C}_{18}-\mathrm{H}\right), 7.12\left(\mathrm{~d}, J=8.2 \mathrm{~Hz}, 1 \mathrm{H}, \mathrm{C}_{19}-\mathrm{H}\right), 6.95(\mathrm{t}, J=7.6 \mathrm{~Hz}, 1 \mathrm{H}$, $\left.\mathrm{C}_{17}-\mathrm{H}\right), 4.71\left(\mathrm{td}, \mathrm{J}=10.9,4.4 \mathrm{~Hz}, 1 \mathrm{H}, \mathrm{C}_{1}-\mathrm{H}\right), 4.07\left(\mathrm{~s}, 2 \mathrm{H}, \mathrm{C}_{12}-\mathrm{H}\right), 3.86\left(\mathrm{~s}, 3 \mathrm{H}, \mathrm{C}_{21}-\mathrm{H}\right), 1.96$ $\left(\mathrm{dtd}, J=12.1,4.2,1.8 \mathrm{~Hz}, 1 \mathrm{H}, \mathrm{C}_{6}-\mathrm{H}_{\mathrm{a}}\right), 1.81\left(\mathrm{qt}, J=7.0,3.5 \mathrm{~Hz}, 1 \mathrm{H}, \mathrm{C}_{7}-\mathrm{H}\right), 1.67(\mathrm{q}, J=2.6$, $\left.1.9 \mathrm{~Hz}, 1 \mathrm{H}, \mathrm{C}_{4}-\mathrm{H}_{\mathrm{a}}\right), 1.64\left(\mathrm{t}, J=2.9 \mathrm{~Hz}, 1 \mathrm{H}, \mathrm{C}_{3}-\mathrm{H}_{\mathrm{a}}\right), 1.45(\mathrm{dddt}, J=12.2,9.4,6.6,3.1 \mathrm{~Hz}, 1 \mathrm{H}$, $\left.\mathrm{C}_{5}-\mathrm{H}\right), 1.37\left(\mathrm{ddt}, J=14.2,11.0,3.1 \mathrm{~Hz}, 1 \mathrm{H}, \mathrm{C}_{2}-\mathrm{H}\right), 1.07-1.00\left(\mathrm{~m}, 1 \mathrm{H}, \mathrm{C}_{3}-\mathrm{H}_{\mathrm{b}}\right), 1.00-0.94(\mathrm{~m}$, $\left.1 \mathrm{H}, \mathrm{C}_{6}-\mathrm{H}_{\mathrm{b}}\right), 0.88\left(\mathrm{~d}, J=6.6 \mathrm{~Hz}, 3 \mathrm{H}, \mathrm{C}_{10}-\mathrm{H}\right), 0.86\left(\mathrm{~d}, J=7.0 \mathrm{~Hz}, 3 \mathrm{H}, \mathrm{C}_{8}-\mathrm{H}\right.$ or $\left.\mathrm{C}_{9}-\mathrm{H}\right), 0.85-0.80$ $\left(\mathrm{m}, 1 \mathrm{H}, \mathrm{C}_{4}-\mathrm{H}_{\mathrm{b}}\right), 0.71\left(\mathrm{~d}, J=7.0 \mathrm{~Hz}, 3 \mathrm{H}, \mathrm{C}_{8}-\mathrm{H}\right.$ or $\left.\mathrm{C}_{9}-\mathrm{H}\right) ;{ }^{13} \mathrm{C}$ NMR $\left(125 \mathrm{MHz}, \mathrm{CDCl}_{3}\right) \delta$ $167.77,157.57,154.11,151.18,131.67,125.49,119.10,118.15,110.96,76.54,46.92,40.63,35.77$, 34.08, 33.40, 31.40, 26.14, 23.31, 21.94), 20.72, 16.22; ESI-MS m/z: $404.20[\mathrm{M}+\mathrm{H}]^{+}$. Anal. Calcd. for $\mathrm{C}_{21} \mathrm{H}_{29} \mathrm{~N}_{3} \mathrm{O}_{3} \mathrm{~S}: \mathrm{C}, 62.50 ; \mathrm{H}, 7.24 ; \mathrm{N}, 10.41$; Found: C, 62.48; H, 7.20; N, 10.39.

Compound (5r): (1R,2S,5R)-2-isopropyl-5-methylcyclohexyl-2-((5-(4-hydroxyphenyl)-4methyl-4H-1,2,4-triazol-3-yl)thio)acetate. White solid. Yield: 76.0\%, m.p. 137.3-138.2 ${ }^{\circ} \mathrm{C}$; IR $\left(\mathrm{KBr}, \mathrm{cm}^{-1}\right)$ : 3435(w, O-H), 2953, 2929, 2866 (s, C-H), 1741 (s, C=O), 1476 (s, C=N), 698 (w, C-S-C); ${ }^{1} \mathrm{H}$ NMR (500 MHz, CDCl $) \delta 10.26(\mathrm{~s}, 1 \mathrm{H}, \mathrm{OH}-\mathrm{H}), 7.35\left(\mathrm{~d}, J=8.6 \mathrm{~Hz}, 2 \mathrm{H}, \mathrm{C}_{16}-\mathrm{H}\right.$, $\left.\mathrm{C}_{20}-\mathrm{H}\right), 6.91\left(\mathrm{~d}, \mathrm{~J}=8.6 \mathrm{~Hz}, 2 \mathrm{H}, \mathrm{C}_{17}-\mathrm{H}, \mathrm{C}_{19}-\mathrm{H}\right), 4.71\left(\mathrm{td}, J=10.9,4.4 \mathrm{~Hz}, 1 \mathrm{H}, \mathrm{C}_{1}-\mathrm{H}\right), 4.06(\mathrm{~s}$, $\left.2 \mathrm{H}, \mathrm{C}_{12}-\mathrm{H}\right), 3.62\left(\mathrm{~s}, 3 \mathrm{H}, \mathrm{C}_{21}-\mathrm{H}\right), 1.97\left(\mathrm{~d}, J=12.2 \mathrm{~Hz}, 1 \mathrm{H}, \mathrm{C}_{6}-\mathrm{H}_{\mathrm{a}}\right), 1.81(\mathrm{td}, J=7.0,2.7 \mathrm{~Hz}, 1 \mathrm{H}$, $\left.\mathrm{C}_{7}-\mathrm{H}\right), 1.66\left(\mathrm{~d}, J=3.3 \mathrm{~Hz}, 1 \mathrm{H}, \mathrm{C}_{4}-\mathrm{H}_{\mathrm{a}}\right), 1.63\left(\mathrm{q}, J=2.8 \mathrm{~Hz}, 1 \mathrm{H}, \mathrm{C}_{3}-\mathrm{H}_{\mathrm{a}}\right), 1.44(\mathrm{ddt}, J=15.3$, 8.8, 3.4 Hz, $\left.1 \mathrm{H}, \mathrm{C}_{5}-\mathrm{H}\right), 1.40-1.34\left(\mathrm{~m}, 1 \mathrm{H}, \mathrm{C}_{2}-\mathrm{H}\right), 1.07-1.00\left(\mathrm{~m}, 1 \mathrm{H}, \mathrm{C}_{3}-\mathrm{H}_{\mathrm{b}}\right), 1.00-0.93(\mathrm{~m}, 1 \mathrm{H}$, $\left.\mathrm{C}_{6}-\mathrm{H}_{\mathrm{b}}\right), 0.86\left(\mathrm{t}, J=6.7 \mathrm{~Hz}, 6 \mathrm{H}, \mathrm{C}_{10}-\mathrm{H}, \mathrm{C}_{8}-\mathrm{H}\right.$ or $\left.\mathrm{C}_{9}-\mathrm{H}\right), 0.81\left(\mathrm{dd}, J=12.5,3.4 \mathrm{~Hz}, 1 \mathrm{H}, \mathrm{C}_{4}-\mathrm{H}_{\mathrm{b}}\right)$, $0.71\left(\mathrm{~d}, J=6.9 \mathrm{~Hz}, 3 \mathrm{H}, \mathrm{C}_{8}-\mathrm{H}\right.$ or $\left.\mathrm{C}_{9}-\mathrm{H}\right) ;{ }^{13} \mathrm{C}$ NMR $\left(125 \mathrm{MHz}, \mathrm{CDCl}_{3}\right) \delta 168.02,159.76,156.50$, $150.24,130.02,116.50,116.42,76.49,46.91,40.61,35.87,34.09,31.86,31.40,26.18,23.34,21.95$, 20.72, 16.25; ESI-MS $m / z: 404.19[\mathrm{M}+\mathrm{H}]^{+}$. Anal. Calcd. for $\mathrm{C}_{21} \mathrm{H}_{29} \mathrm{~N}_{3} \mathrm{O}_{3} \mathrm{~S}: \mathrm{C}, 62.50 ; \mathrm{H}$, 7.24; N,10.41; Found: C, 62.48; H, 7.22; N, 10.40 .

Compound (5s): (1R,2S,5R)-2-isopropyl-5-methylcyclohexyl-2-((5-(2-aminophenyl)-4-methyl4H-1,2,4-triazol-3-yl)thio) acetate. Pale yellow liquid. Yield: 59.6\%; $\mathrm{IR}\left(\mathrm{KBr}, \mathrm{cm}^{-1}\right): 3454,3351$ (m, N-H), 2954, 2928, 2869 (s, C-H), 1729 (s, C=O), 1469 (s, C=N), 707 (w, C-S-C); ${ }^{1}$ H NMR $\left(500 \mathrm{MHz}, \mathrm{CDCl}_{3}\right) \delta 7.22\left(\mathrm{td}, J=7.9,1.5 \mathrm{~Hz}, 1 \mathrm{H}, \mathrm{C}_{20}-\mathrm{H}\right), 7.17\left(\mathrm{dd}, J=7.7,1.5 \mathrm{~Hz}, 1 \mathrm{H}, \mathrm{C}_{18}-\mathrm{H}\right)$, 6.82-6.76 (m, 2H, $\left.\mathrm{C}_{17}-\mathrm{H}, \mathrm{C}_{19}-\mathrm{H}\right), 4.84\left(\mathrm{~s}, 2 \mathrm{H}, \mathrm{NH}_{2}-\mathrm{H}\right), 4.72\left(\mathrm{td}, J=10.9,4.4 \mathrm{~Hz}, 1 \mathrm{H}, \mathrm{C}_{1}-\mathrm{H}\right)$, $4.09\left(\mathrm{~s}, 2 \mathrm{H}, \mathrm{C}_{12}-\mathrm{H}\right), 3.61\left(\mathrm{~s}, 3 \mathrm{H}, \mathrm{C}_{21}-\mathrm{H}\right), 1.99\left(\mathrm{dtd}, J=12.1,4.1,1.7 \mathrm{~Hz}, 1 \mathrm{H}, \mathrm{C}_{6}-\mathrm{H}_{\mathrm{a}}\right), 1.85(\mathrm{pd}$, $\left.J=7.0,2.8 \mathrm{~Hz}, 1 \mathrm{H}, \mathrm{C}_{7}-\mathrm{H}\right), 1.68\left(\mathrm{q}, J=2.7,2.1 \mathrm{~Hz}, 1 \mathrm{H}, \mathrm{C}_{4}-\mathrm{H}_{\mathrm{a}}\right), 1.65\left(\mathrm{q}, J=2.8 \mathrm{~Hz}, 1 \mathrm{H}, \mathrm{C}_{3}-\mathrm{H}_{\mathrm{a}}\right)$, 1.46 (dddd, $\left.J=12.1,8.9,6.2,3.2 \mathrm{~Hz}, 1 \mathrm{H}, \mathrm{C}_{5}-\mathrm{H}\right), 1.39\left(\mathrm{ddt}, J=14.2,10.9,3.1 \mathrm{~Hz}, 1 \mathrm{H}, \mathrm{C}_{2}-\mathrm{H}\right)$, $1.04\left(\mathrm{ddd}, J=15.7,9.7,3.1 \mathrm{~Hz}, 1 \mathrm{H}, \mathrm{C}_{3}-\mathrm{H}_{\mathrm{b}}\right), 1.01-0.95\left(\mathrm{~m}, 1 \mathrm{H}, \mathrm{C}_{6}-\mathrm{H}_{\mathrm{b}}\right), 0.88(\mathrm{t}, J=6.5 \mathrm{~Hz}, 6 \mathrm{H}$, $\mathrm{C}_{10}-\mathrm{H}, \mathrm{C}_{8}-\mathrm{H}$ or $\left.\mathrm{C}_{9}-\mathrm{H}\right), 0.83\left(\mathrm{dd}, J=12.3,3.3 \mathrm{~Hz}, 1 \mathrm{H}, \mathrm{C}_{4}-\mathrm{H}_{\mathrm{b}}\right), 0.73\left(\mathrm{~d}, J=6.9 \mathrm{~Hz}, 3 \mathrm{H}, \mathrm{C}_{8}-\mathrm{H}\right.$ or $\left.\mathrm{C}_{9}-\mathrm{H}\right) ;{ }^{13} \mathrm{C}$ NMR $\left(125 \mathrm{MHz}, \mathrm{CDCl}_{3}\right) \delta 168.01,154.57,150.25,146.68,131.11,129.14,117.40$, 116.59, 110.43, 76.39, 46.93, 40.62, 35.68, 34.11, 32.04, 31.41, 26.19, 23.37, 21.96, 20.74, 16.28; ESI-MS $m / z: 403.23[\mathrm{M}+\mathrm{H}]^{+}$. Anal. Calcd. for $\mathrm{C}_{21} \mathrm{H}_{30} \mathrm{~N}_{4} \mathrm{O}_{2} \mathrm{~S}: \mathrm{C}, 62.66 ; \mathrm{H}, 7.51 ; \mathrm{N}, 13.92$; Found: C, 62.65; H, 7.50; N, 13.90 .

Compound (5t): (1R,2S,5R)-2-isopropyl-5-methylcyclohexyl-2-((5-(2-aminophenyl)-4-methyl4H-1,2,4-triazol-3-yl)thio)acetate. Pale yellow liquid. Yield: $52.7 \%$; IR $\left(\mathrm{KBr}, \mathrm{cm}^{-1}\right): 3452,3348$ (m, N-H), 2954, 2927, 2868 (s, C-H), 1728 (s, C=O), 1469 (s, C=N), 702 (w, C-S-C); ${ }^{1} \mathrm{H}$ NMR $\left(500 \mathrm{MHz}, \mathrm{CDCl}_{3}\right) \delta 7.40\left(\mathrm{~d}, J=7.9 \mathrm{~Hz}, 2 \mathrm{H}, \mathrm{C}_{16}-\mathrm{H}, \mathrm{C}_{20}-\mathrm{H}\right), 6.74\left(\mathrm{~d}, J=8.1 \mathrm{~Hz}, 2 \mathrm{H}, \mathrm{C}_{17}-\mathrm{H}\right.$, $\left.\mathrm{C}_{19}-\mathrm{H}\right), 4.70\left(\mathrm{td}, J=10.9,4.4 \mathrm{~Hz}, 1 \mathrm{H}, \mathrm{C}_{1}-\mathrm{H}\right), 4.04\left(\mathrm{~s}, 2 \mathrm{H}, \mathrm{C}_{12}-\mathrm{H}\right), 3.65-3.59\left(\mathrm{~m}, 3 \mathrm{H}, \mathrm{C}_{21}-\mathrm{H}\right)$, $2.95\left(\mathrm{~s}, 2 \mathrm{H}, \mathrm{NH}_{2}-\mathrm{H}\right), 2.00-1.94\left(\mathrm{~m}, 1 \mathrm{H}, \mathrm{C}_{6}-\mathrm{H}_{\mathrm{a}}\right), 1.81\left(\mathrm{td}, J=7.0,2.7 \mathrm{~Hz}, 1 \mathrm{H}, \mathrm{C}_{7}-\mathrm{H}\right), 1.67(\mathrm{~d}$, $\left.J=3.3 \mathrm{~Hz}, 1 \mathrm{H}, \mathrm{C}_{4}-\mathrm{H}_{\mathrm{a}}\right), 1.64\left(\mathrm{q}, J=2.9 \mathrm{~Hz}, 1 \mathrm{H}, \mathrm{C}_{3}-\mathrm{H}_{\mathrm{a}}\right), 1.45(\mathrm{dddt}, J=12.0,8.8,6.2,3.4 \mathrm{~Hz}$, $\left.1 \mathrm{H}, \mathrm{C}_{5}-\mathrm{H}\right), 1.37\left(\mathrm{ddt}, J=14.4,10.9,2.8 \mathrm{~Hz}, 1 \mathrm{H}, \mathrm{C}_{2}-\mathrm{H}\right), 1.08-1.00\left(\mathrm{~m}, 1 \mathrm{H}, \mathrm{C}_{3}-\mathrm{H}_{\mathrm{b}}\right), 1.00-0.93$ $\left(\mathrm{m}, 1 \mathrm{H}, \mathrm{C}_{6}-\mathrm{H}_{\mathrm{b}}\right), 0.90-0.85\left(\mathrm{~m}, 6 \mathrm{H}, \mathrm{C}_{10}-\mathrm{H}, \mathrm{C}_{8}-\mathrm{H}\right.$ or $\left.\mathrm{C}_{9}-\mathrm{H}\right), 0.85-0.79\left(\mathrm{~m}, 1 \mathrm{H}, \mathrm{C}_{4}-\mathrm{H}_{\mathrm{b}}\right), 0.72(\mathrm{~d}$, $J=7.0 \mathrm{~Hz}, 3 \mathrm{H}, \mathrm{C}_{8}-\mathrm{H}$ or $\left.\mathrm{C}_{9}-\mathrm{H}\right) ;{ }^{13} \mathrm{C}$ NMR $\left(125 \mathrm{MHz}, \mathrm{CDCl}_{3}\right) \delta 168.17,156.46,149.75,148.34$, $129.88,116.36,114.84,76.31,46.91,40.62,36.12,34.11,31.85,31.40,26.16,23.35,21.97,20.75$, 16.27; ESI-MS $m / z: 403.23[\mathrm{M}+\mathrm{H}]^{+}$. Anal. Calcd. for $\mathrm{C}_{21} \mathrm{H}_{30} \mathrm{~N}_{4} \mathrm{O}_{2} \mathrm{~S}: \mathrm{C}, 62.66 ; \mathrm{H}, 7.51 ; \mathrm{N}$, 13.92; Found: C, 62.64; H, 7.49; N, 13.91. 
Compound (5u): (1R,2S,5R)-2-isopropyl-5-methylcyclohexyl-2-((5-(4-(tert-butyl)phenyl)4-methyl-4H-1,2,4-triazol-3-yl)thio)acetate. White solid. Yield: $66.1 \%$, m.p. $100.3-102.6{ }^{\circ} \mathrm{C}$; IR $\left(\mathrm{KBr}, \mathrm{cm}^{-1}\right)$ : 2955, 2926, 2871 (s, C-H), 1743(s, C=O), 1468 (s, C=N), 704 (w, C-S-C); ${ }^{1} \mathrm{H}$ NMR $\left(500 \mathrm{MHz}, \mathrm{CDCl}_{3}\right) \delta$ 7.58-7.54 (m, 2H, C $\left.16-\mathrm{H}, \mathrm{C}_{20}-\mathrm{H}\right), 7.53-7.49\left(\mathrm{~m}, 2 \mathrm{H}, \mathrm{C}_{17}-\mathrm{H}, \mathrm{C}_{19}-\mathrm{H}\right), 4.71$ $\left(\mathrm{td}, J=10.9,4.4 \mathrm{~Hz}, 1 \mathrm{H}, \mathrm{C}_{1}-\mathrm{H}\right), 4.08\left(\mathrm{~s}, 2 \mathrm{H}, \mathrm{C}_{12}-\mathrm{H}\right), 3.66\left(\mathrm{~s}, 3 \mathrm{H}, \mathrm{C}_{21}-\mathrm{H}\right), 1.98$ (dtd, J = 12.0, 4.0, $\left.1.7 \mathrm{~Hz}, 1 \mathrm{H}, \mathrm{C}_{6}-\mathrm{H}_{\mathrm{a}}\right), 1.84\left(\mathrm{dd}, J=7.0,2.8 \mathrm{~Hz}, 1 \mathrm{H}, \mathrm{C}_{7}-\mathrm{H}\right), 1.68(\mathrm{q}, J=2.6,1.8 \mathrm{~Hz}, 1 \mathrm{H}$, $\left.\mathrm{C}_{4}-\mathrm{H}_{\mathrm{a}}\right), 1.65\left(\mathrm{q}, J=2.8 \mathrm{~Hz}, 1 \mathrm{H}, \mathrm{C}_{3}-\mathrm{H}_{\mathrm{a}}\right), 1.46\left(\mathrm{tdd}, J=8.7,5.9,3.1 \mathrm{~Hz}, 1 \mathrm{H}, \mathrm{C}_{5}-\mathrm{H}\right), 1.43-1.36(\mathrm{~m}$, $\left.1 \mathrm{H}, \mathrm{C}_{2}-\mathrm{H}\right), 1.35\left(\mathrm{~s}, 9 \mathrm{H}, \mathrm{C}_{23}-\mathrm{H}, \mathrm{C}_{24}-\mathrm{H}, \mathrm{C}_{25}-\mathrm{H}\right), 1.08-1.01\left(\mathrm{~m}, 1 \mathrm{H}, \mathrm{C}_{3}-\mathrm{H}_{\mathrm{b}}\right), 1.01-0.95(\mathrm{~m}, 1 \mathrm{H}$, $\left.\mathrm{C}_{6}-\mathrm{H}_{\mathrm{b}}\right), 0.88\left(\mathrm{t}, J=7.1 \mathrm{~Hz}, 6 \mathrm{H}, \mathrm{C}_{10}-\mathrm{H}, \mathrm{C}_{8}-\mathrm{H}\right.$ or $\left.\mathrm{C}_{9}-\mathrm{H}\right), 0.86\left(\mathrm{~s}, 1 \mathrm{H}, \mathrm{C}_{4}-\mathrm{H}_{\mathrm{b}}\right), 0.73(\mathrm{~d}, J=7.0 \mathrm{~Hz}$, $3 \mathrm{H}, \mathrm{C}_{8}-\mathrm{H}$ or $\left.\mathrm{C}_{9}-\mathrm{H}\right) ;{ }^{13} \mathrm{C}$ NMR $\left(125 \mathrm{MHz}, \mathrm{CDCl}_{3}\right) \delta 168.23,156.28,153.58,150.41,128.44$, $126.01,124.14,76.46,47.05,40.76,36.15,35.02,34.24,31.97,31.54,31.32,26.30,23.49,22.09$, 20.88, 16.40; ESI-MS m/z: $444.28[\mathrm{M}+\mathrm{H}]^{+}$. Anal. Calcd. for $\mathrm{C}_{25} \mathrm{H}_{37} \mathrm{~N}_{3} \mathrm{O}_{2} \mathrm{~S}: \mathrm{C}, 67.68 ; \mathrm{H}$, 8.41; N, 9.47; Found: C, 67.66; H, 8.40; N, 9.46.

Compound (5v): (1R,2S,5R)-2-isopropyl-5-methylcyclohexyl-2-((5-(3,4-dimethoxyphenyl)4-methyl-4H-1,2,4-triazol-3-yl)thio)acetate. Pale yellow liquid. Yield: $50.9 \%$; IR (KBr, $\left.\mathrm{cm}^{-1}\right)$ : 2955, 2926, 2869 (s, C-H), 1731 (s, C=O), 1422 (s, C=N), 686 (w, C-S-C); ${ }^{1} \mathrm{H}$ NMR (500 MHz, $\left.\mathrm{CDCl}_{3}\right) \delta 7.23\left(\mathrm{~d}, \mathrm{~J}=2.0 \mathrm{~Hz}, 1 \mathrm{H}, \mathrm{C}_{20}-\mathrm{H}\right), 7.10\left(\mathrm{dd}, \mathrm{J}=8.3,2.0 \mathrm{~Hz}, 1 \mathrm{H}, \mathrm{C}_{16}-\mathrm{H}\right), 6.95(\mathrm{~d}$, $\left.J=8.3 \mathrm{~Hz}, 1 \mathrm{H}, \mathrm{C}_{19}-\mathrm{H}\right), 4.70\left(\mathrm{td}, J=10.9,4.4 \mathrm{~Hz}, 1 \mathrm{H}, \mathrm{C}_{1}-\mathrm{H}\right), 4.06\left(\mathrm{~s}, 2 \mathrm{H}, \mathrm{C}_{12}-\mathrm{H}\right), 3.92(\mathrm{~d}$, $\left.J=6.4 \mathrm{~Hz}, 6 \mathrm{H}, \mathrm{C}_{22}-\mathrm{H}, \mathrm{C}_{23}-\mathrm{H}\right), 3.66\left(\mathrm{~s}, 3 \mathrm{H}, \mathrm{C}_{21}-\mathrm{H}\right), 1.97\left(\mathrm{dd}, J=11.9,4.5 \mathrm{~Hz}, 1 \mathrm{H}, \mathrm{C}_{6}-\mathrm{H}_{\mathrm{a}}\right)$, $1.81\left(\mathrm{ddd}, J=14.0,7.0,2.8 \mathrm{~Hz}, 1 \mathrm{H}, \mathrm{C}_{7}-\mathrm{H}\right), 1.69-1.66\left(\mathrm{~m}, 1 \mathrm{H}, \mathrm{C}_{4}-\mathrm{H}_{\mathrm{a}}\right), 1.64(\mathrm{q}, J=2.8 \mathrm{~Hz}, 1 \mathrm{H}$, $\left.\mathrm{C}_{3}-\mathrm{H}_{\mathrm{a}}\right), 1.45\left(\mathrm{dddd}, J=12.1,8.9,6.9,3.1 \mathrm{~Hz}, 1 \mathrm{H}, \mathrm{C}_{5}-\mathrm{H}\right), 1.37(\mathrm{ddt}, J=14.2,10.9,3.1 \mathrm{~Hz}, 1 \mathrm{H}$, $\left.\mathrm{C}_{2}-\mathrm{H}\right), 1.08-1.00\left(\mathrm{~m}, 1 \mathrm{H}, \mathrm{C}_{3}-\mathrm{H}_{\mathrm{b}}\right), 1.00-0.94\left(\mathrm{~m}, 1 \mathrm{H}, \mathrm{C}_{6}-\mathrm{H}_{\mathrm{b}}\right), 0.87\left(\mathrm{t}, J=7.0 \mathrm{~Hz}, 6 \mathrm{H}, \mathrm{C}_{10}-\mathrm{H}\right.$, $\mathrm{C}_{8}-\mathrm{H}$ or $\left.\mathrm{C}_{9}-\mathrm{H}\right), 0.85-0.80\left(\mathrm{~m}, 1 \mathrm{H}, \mathrm{C}_{4}-\mathrm{H}_{\mathrm{b}}\right), 0.72\left(\mathrm{~d}, \mathrm{~J}=7.0 \mathrm{~Hz}, 3 \mathrm{H}, \mathrm{C}_{8}-\mathrm{H}\right.$ or $\left.\mathrm{C}_{9}-\mathrm{H}\right) ;{ }^{13} \mathrm{C}$ NMR $\left(125 \mathrm{MHz}_{\mathrm{CDCl}}\right) \delta 168.11,156.00,150.62,150.28,149.34,120.98,119.48,112.01,110.99$, 76.35, 56.09, 56.01, 46.93, 40.64, 36.08, 34.11, 31.93, 31.41, 26.17, 23.35, 21.97, 20.75, 16.28; ESI-MS m/z: $448.25[\mathrm{M}+\mathrm{H}]^{+}$. Anal. Calcd. for $\mathrm{C}_{23} \mathrm{H}_{33} \mathrm{~N}_{3} \mathrm{O}_{4} \mathrm{~S}: \mathrm{C}, 61.72 ; \mathrm{H}, 7.43 ; \mathrm{N}, 9.39$; Found: C, 61.70; H, 7.42; N, 9.36.

Compound (5w): (1R,2S,5R)-2-isopropyl-5-methylcyclohexyl-2-((5-(3,5-dimethoxyphenyl)4-methyl-4H-1,2,4-triazol-3-yl)thio)acetate. Pale yellow liquid. Yield: $62.7 \%$; IR (KBr, $\left.\mathrm{cm}^{-1}\right)$ : 2954, 2926, 2869 (s, C-H), 1731 (s, C=O), 1474 (s, C=N), 713 (w, C-S-C); ${ }^{1} \mathrm{H}$ NMR (500 MHz, $\left.\mathrm{CDCl}_{3}\right) 6.75\left(\mathrm{~d}, \mathrm{~J}=2.3 \mathrm{~Hz}, 2 \mathrm{H}, \mathrm{C}_{16}-\mathrm{H}, \mathrm{C}_{20}-\mathrm{H}\right), 6.57\left(\mathrm{t}, \mathrm{J}=2.3 \mathrm{~Hz}, 1 \mathrm{H}, \mathrm{C}_{18}-\mathrm{H}\right), 4.71(\mathrm{td}$, $\left.J=10.9,4.4 \mathrm{~Hz}, 1 \mathrm{H}, \mathrm{C}_{1}-\mathrm{H}\right), 4.07\left(\mathrm{~d}, J=1.3 \mathrm{~Hz}, 2 \mathrm{H}, \mathrm{C}_{12}-\mathrm{H}\right), 3.82\left(\mathrm{~s}, 6 \mathrm{H}, \mathrm{C}_{22}-\mathrm{H}, \mathrm{C}_{23}-\mathrm{H}\right), 3.66$ $\left(\mathrm{s}, 3 \mathrm{H}, \mathrm{C}_{21}-\mathrm{H}\right), 1.98\left(\mathrm{dtd}, J=12.0,4.0,1.7 \mathrm{~Hz}, 1 \mathrm{H}, \mathrm{C}_{6}-\mathrm{H}_{\mathrm{a}}\right), 1.82(\mathrm{ddd}, J=11.2,7.0,3.5 \mathrm{~Hz}, 1 \mathrm{H}$, $\left.\mathrm{C}_{7}-\mathrm{H}\right), 1.67\left(\mathrm{q}, J=2.6,1.8 \mathrm{~Hz}, 1 \mathrm{H}, \mathrm{C}_{4}-\mathrm{H}_{\mathrm{a}}\right), 1.66-1.62\left(\mathrm{~m}, 1 \mathrm{H}, \mathrm{C}_{3}-\mathrm{H}_{\mathrm{a}}\right), 1.46(\mathrm{dddt}, J=12.1,8.9$, $\left.6.2,3.4 \mathrm{~Hz}, 1 \mathrm{H}, \mathrm{C}_{5}-\mathrm{H}\right), 1.41-1.34\left(\mathrm{~m}, 1 \mathrm{H}, \mathrm{C}_{2}-\mathrm{H}\right), 1.08-1.01\left(\mathrm{~m}, 1 \mathrm{H}, \mathrm{C}_{3}-\mathrm{H}_{\mathrm{b}}\right), 1.00-0.95(\mathrm{~m}, 1 \mathrm{H}$, $\left.\mathrm{C}_{6}-\mathrm{H}_{\mathrm{b}}\right), 0.90-0.86\left(\mathrm{~m}, 6 \mathrm{H}, \mathrm{C}_{10}-\mathrm{H}, \mathrm{C}_{8}-\mathrm{H}\right.$ or $\left.\mathrm{C}_{9}-\mathrm{H}\right), 0.85-0.81\left(\mathrm{~m}, 1 \mathrm{H}, \mathrm{C}_{4}-\mathrm{H}_{\mathrm{b}}\right), 0.72(\mathrm{~d}, J=7.0$ $\mathrm{Hz}, 3 \mathrm{H}, \mathrm{C}_{8}-\mathrm{H}$ or $\left.\mathrm{C}_{9}-\mathrm{H}\right) ;{ }^{13} \mathrm{C}$ NMR $\left(125 \mathrm{MHz}, \mathrm{CDCl}_{3}\right) \delta 168.16,161.15,156.06,150.76,128.66$, 106.82, 102.30, 76.49, 55.70, 47.04, 40.75, 36.10, 34.23, 32.06, 31.52, 26.29, 23.47, 22.08, 20.87, 16.39; ESI-MS m/z: $448.24[\mathrm{M}+\mathrm{H}]^{+}$. Anal. Calcd. for $\mathrm{C}_{23} \mathrm{H}_{33} \mathrm{~N}_{3} \mathrm{O}_{4} \mathrm{~S}: \mathrm{C}, 61.72 ; \mathrm{H}, 7.43 ; \mathrm{N}$, 9.39; Found: C, 61.71; H, 7.40; N, 9.37.

Compound (5x): (1R,2S,5R)-2-isopropyl-5-methylcyclohexyl-2-((5-(furan-2-yl)-4-methyl4H-1,2,4-triazol-3-yl)thio)acetate. White solid. Yield: $51.3 \%$, m.p. $136.2-137.7^{\circ} \mathrm{C}$; IR (KBr, $\mathrm{cm}^{-1}$ ): 2961, 2935, 2866 (s, C-H), 1734 (s, C=O), 1474 (s, C=N), 707 (w, C-S-C); ${ }^{1} \mathrm{H}$ NMR $\left(500 \mathrm{MHz} \mathrm{CDCl}_{3}\right) \delta 7.58\left(\mathrm{t}, J=1.2 \mathrm{~Hz}, 1 \mathrm{H}, \mathrm{C}_{18}-\mathrm{H}\right), 7.05\left(\mathrm{~d}, J=3.5 \mathrm{~Hz}, 1 \mathrm{H}, \mathrm{C}_{16}-\mathrm{H}\right), 6.56$ $\left(\mathrm{dd}, J=3.5,1.8 \mathrm{~Hz}, 1 \mathrm{H}, \mathrm{C}_{17}-\mathrm{H}\right), 4.69\left(\mathrm{td}, J=10.9,4.4 \mathrm{~Hz}, 1 \mathrm{H}, \mathrm{C}_{1}-\mathrm{H}\right), 4.03(\mathrm{~d}, J=2.3 \mathrm{~Hz}, 2 \mathrm{H}$, $\left.\mathrm{C}_{12}-\mathrm{H}\right), 3.83\left(\mathrm{~s}, 3 \mathrm{H}, \mathrm{C}_{19}-\mathrm{H}\right), 1.95\left(\mathrm{dd}, J=10.8,6.0 \mathrm{~Hz}, 1 \mathrm{H}, \mathrm{C}_{6}-\mathrm{H}_{\mathrm{a}}\right), 1.78(\mathrm{td}, J=6.9,2.8 \mathrm{~Hz}$, $\left.1 \mathrm{H}, \mathrm{C}_{7}-\mathrm{H}\right), 1.69-1.66\left(\mathrm{~m}, 1 \mathrm{H}, \mathrm{C}_{4}-\mathrm{H}_{\mathrm{a}}\right), 1.64\left(\mathrm{q}, J=2.9 \mathrm{~Hz}, 1 \mathrm{H}, \mathrm{C}_{3}-\mathrm{H}_{\mathrm{a}}\right), 1.44(\mathrm{dddd}, J=13.0$, 9.9, 6.6, 3.6 Hz, $\left.1 \mathrm{H}, \mathrm{C}_{5}-\mathrm{H}\right), 1.40-1.32\left(\mathrm{~m}, 1 \mathrm{H}, \mathrm{C}_{2}-\mathrm{H}\right), 1.07-0.99\left(\mathrm{~m}, 1 \mathrm{H}, \mathrm{C}_{3}-\mathrm{H}_{\mathrm{b}}\right), 0.99-0.93(\mathrm{~m}$, $\left.1 \mathrm{H}, \mathrm{C}_{6}-\mathrm{H}_{\mathrm{b}}\right), 0.86\left(\mathrm{dd}, \mathrm{J}=11.1,6.8 \mathrm{~Hz}, 6 \mathrm{H}, \mathrm{C}_{10}-\mathrm{H}, \mathrm{C}_{8}-\mathrm{H}\right.$ or $\left.\mathrm{C}_{9}-\mathrm{H}\right), 0.84-0.79\left(\mathrm{~m}, 1 \mathrm{H}, \mathrm{C}_{4}-\mathrm{H}_{\mathrm{b}}\right)$, $0.71\left(\mathrm{~d}, J=6.9 \mathrm{~Hz}, 3 \mathrm{H}, \mathrm{C}_{8}-\mathrm{H}\right.$ or $\left.\mathrm{C}_{9}-\mathrm{H}\right) ;{ }^{13} \mathrm{C} \mathrm{NMR}\left(125 \mathrm{MHz} \mathrm{CDCl}_{3}\right) \delta 168.10,150.36,148.42$, 144.06, 142.43, 111.97, 111.91, 76.48, 47.02, 40.72, 36.35, 34.22, 32.08, 31.52, 26.26, 23.45, 22.06, 20.84, 16.35; ESI-MS m/z: $378.19[\mathrm{M}+\mathrm{H}]^{+}$. Anal. Calcd. for $\mathrm{C}_{19} \mathrm{H}_{27} \mathrm{~N}_{3} \mathrm{O}_{3} \mathrm{~S}: \mathrm{C}, 60.45 ; \mathrm{H}$, 7.21; N, 11.13; Found: C, 60.43; H, 7.20; N, 11.12. 
Compound (5y): (1R,2S,5R)-2-isopropyl-5-methylcyclohexyl-2-((4-methyl-5-(thiophen-2yl)-4H-1,2,4-triazol-3-yl)thio)acetate. White solid. Yield: $62.5 \%$, m.p. $127.2-127.9{ }^{\circ} \mathrm{C}$; IR (KBr, $\mathrm{cm}^{-1}$ ): 2952, 2934, 2915, 2864 (s, C-H), 1736 (s, C=O), 1474 (s, C=N), 707 (w, C-S-C); ${ }^{1} \mathrm{H}$ NMR $\left(500 \mathrm{MHz}, \mathrm{CDCl}_{3}\right) \delta 7.49\left(\mathrm{dd}, J=5.1,1.1 \mathrm{~Hz}, 1 \mathrm{H}, \mathrm{C}_{18}-\mathrm{H}\right), 7.46(\mathrm{dd}, J=3.7,1.1 \mathrm{~Hz}, 1 \mathrm{H}$, $\left.\mathrm{C}_{16}-\mathrm{H}\right), 7.16\left(\mathrm{dd}, J=5.1,3.7 \mathrm{~Hz}, 1 \mathrm{H}, \mathrm{C}_{17}-\mathrm{H}\right), 4.69\left(\mathrm{td}, J=10.9,4.4 \mathrm{~Hz}, 1 \mathrm{H}, \mathrm{C}_{1}-\mathrm{H}\right), 4.04(\mathrm{~d}$, $\left.J=2.0 \mathrm{~Hz}, 2 \mathrm{H}, \mathrm{C}_{12}-\mathrm{H}\right), 3.76\left(\mathrm{~s}, 3 \mathrm{H}, \mathrm{C}_{19}-\mathrm{H}\right), 1.96\left(\mathrm{ddq}, J=9.7,3.9,2.0 \mathrm{~Hz}, 1 \mathrm{H}, \mathrm{C}_{6}-\mathrm{H}_{\mathrm{a}}\right), 1.79$ $\left(\mathrm{qd}, J=7.0,2.8 \mathrm{~Hz}, 1 \mathrm{H}, \mathrm{C}_{7}-\mathrm{H}\right), 1.67\left(\mathrm{q}, J=2.6,1.8 \mathrm{~Hz}, 1 \mathrm{H}, \mathrm{C}_{4}-\mathrm{H}_{\mathrm{a}}\right), 1.66-1.62\left(\mathrm{~m}, 1 \mathrm{H}, \mathrm{C}_{3}-\mathrm{H}_{\mathrm{a}}\right)$, 1.45 (tdt, $\left.J=12.2,6.6,3.3 \mathrm{~Hz}, 1 \mathrm{H}, \mathrm{C}_{5}-\mathrm{H}\right), 1.39-1.34\left(\mathrm{~m}, 1 \mathrm{H}, \mathrm{C}_{2}-\mathrm{H}\right), 1.07-0.99\left(\mathrm{~m}, 1 \mathrm{H}, \mathrm{C}_{3}-\mathrm{H}_{\mathrm{b}}\right)$, 0.99-0.93 (m, 1H, C $\left.6-\mathrm{H}_{\mathrm{b}}\right), 0.87\left(\mathrm{dd}, J=9.0,6.7 \mathrm{~Hz}, 6 \mathrm{H}, \mathrm{C}_{10}-\mathrm{H}, \mathrm{C}_{8}-\mathrm{H}\right.$ or $\left.\mathrm{C}_{9}-\mathrm{H}\right), 0.85-0.79$ $\left(\mathrm{m}, 1 \mathrm{H}, \mathrm{C}_{4}-\mathrm{H}_{\mathrm{b}}\right), 0.71\left(\mathrm{~d}, \mathrm{~J}=6.9 \mathrm{~Hz}, 3 \mathrm{H}, \mathrm{C}_{8}-\mathrm{H}\right.$ or $\left.\mathrm{C}_{9}-\mathrm{H}\right) ;{ }^{13} \mathrm{C} \mathrm{NMR}\left(125 \mathrm{MHz} \mathrm{CDCl}_{3}\right) \delta$ 168.13, 151.15, 150.66, 128.49, 128.09, 127.90, 127.86, 76.50, 47.03, 40.73, 36.34, 34.21, 32.05, 31.52, 26.27, 23.45, 22.06, 20.85, 16.37; ESI-MS m/z: $394.16[\mathrm{M}+\mathrm{H}]^{+}$. Anal. Calcd. for $\mathrm{C}_{19} \mathrm{H}_{27} \mathrm{~N}_{3} \mathrm{O}_{2} \mathrm{~S}_{2}$ : C, 57.99; H, 6.92; N,10.68; Found: C, 57.98; H, 6.90; N, 10.66.

Compound (5z): (1R,2S,5R)-2-isopropyl-5-methylcyclohexyl-2-((4-methyl-5-(pyridin-3-yl)4H-1,2,4-triazol-3-yl)thio)acetate. Yellow solid. Yield: $61.5 \%$, m.p. $156.1-158.0{ }^{\circ} \mathrm{C}$; IR (KBr, $\mathrm{cm}^{-1}$ ): 2962, 2945, 2916, 2866 (s, C-H), 1733 (s, C=O), 1481 (s, C=N), 712 (w, C-S-C); ${ }^{1} \mathrm{H}$ NMR $\left(500 \mathrm{MHz}, \mathrm{CDCl}_{3}\right) \delta 8.88\left(\mathrm{dt}, J=2.1,1.1 \mathrm{~Hz}, 1 \mathrm{H}, \mathrm{C}_{19}-\mathrm{H}\right), 8.73(\mathrm{dd}, J=4.1,2.4 \mathrm{~Hz}$, $\left.1 \mathrm{H}, \mathrm{C}_{18}-\mathrm{H}\right), 8.04-7.99\left(\mathrm{~m}, 1 \mathrm{H}, \mathrm{C}_{16}-\mathrm{H}\right), 7.45\left(\mathrm{ddt}, J=7.9,4.9,1.1 \mathrm{~Hz}, 1 \mathrm{H}, \mathrm{C}_{17}-\mathrm{H}\right), 4.71(\mathrm{td}$, $\left.J=11.0,1.3 \mathrm{~Hz}, 1 \mathrm{H}, \mathrm{C}_{1}-\mathrm{H}\right), 4.09\left(\mathrm{~s}, 2 \mathrm{H}, \mathrm{C}_{12}-\mathrm{H}\right), 3.70\left(\mathrm{~s}, 3 \mathrm{H}, \mathrm{C}_{20}-\mathrm{H}\right), 1.97(\mathrm{dt}, J=12.2,4.0 \mathrm{~Hz}$, $\left.1 \mathrm{H}, \mathrm{C}_{6}-\mathrm{H}_{\mathrm{a}}\right), 1.84-1.78\left(\mathrm{~m}, 1 \mathrm{H}, \mathrm{C}_{7}-\mathrm{H}\right), 1.67\left(\mathrm{~d}, J=3.2 \mathrm{~Hz}, 1 \mathrm{H}, \mathrm{C}_{4}-\mathrm{H}_{\mathrm{a}}\right), 1.65(\mathrm{t}, J=2.9 \mathrm{~Hz}, 1 \mathrm{H}$, $\left.\mathrm{C}_{3}-\mathrm{H}_{\mathrm{a}}\right), 1.45\left(\mathrm{dt}, J=15.8,5.3 \mathrm{~Hz}, 1 \mathrm{H}, \mathrm{C}_{5}-\mathrm{H}\right), 1.41-1.34\left(\mathrm{~m}, 1 \mathrm{H}, \mathrm{C}_{2}-\mathrm{H}\right), 1.03$ (ddd, J = 13.5, $\left.10.4,3.3 \mathrm{~Hz}, 1 \mathrm{H}, \mathrm{C}_{3}-\mathrm{H}_{\mathrm{b}}\right), 1.00-0.95\left(\mathrm{~m}, 1 \mathrm{H}, \mathrm{C}_{6}-\mathrm{H}_{\mathrm{b}}\right), 0.87\left(\mathrm{t}, J=7.0 \mathrm{~Hz}, 6 \mathrm{H}, \mathrm{C}_{10}-\mathrm{H}, \mathrm{C}_{8}-\mathrm{H}\right.$ or $\left.\mathrm{C}_{9}-\mathrm{H}\right), 0.85-0.80\left(\mathrm{~m}, 1 \mathrm{H}, \mathrm{C}_{4}-\mathrm{H}_{\mathrm{b}}\right), 0.72\left(\mathrm{~d}, \mathrm{~J}=7.0 \mathrm{~Hz}, 3 \mathrm{H}, \mathrm{C}_{8}-\mathrm{H}\right.$ or $\left.\mathrm{C}_{9}-\mathrm{H}\right) ;{ }^{13} \mathrm{C}$ NMR $(125 \mathrm{MHz}$, $\left.\mathrm{CDCl}_{3}\right) \delta 168.05,153.54,151.60,151.24,148.96,136.26,123.89,123.59,76.60,47.06,40.76$, 36.05, 34.21, 32.06, 31.52, 26.31, 23.47, 22.08, 20.86, 16.40; ESI-MS m/z: $389.19[\mathrm{M}+\mathrm{H}]^{+}$. Anal. Calcd. for $\mathrm{C}_{20} \mathrm{H}_{28} \mathrm{~N}_{4} \mathrm{O}_{2} \mathrm{~S}: \mathrm{C}, 61.83 ; \mathrm{H}, 7.26 ; \mathrm{N}, 14.42$; Found: C, 61.82; H, 7.25; N, 14.40 .

\subsection{Antifungal Activity Test}

The primary biological activity of the menthol-based 1,2,4-triazole-thioether compounds was evaluated by agar dilution method against the tested fungi. The tested compound was dissolved in acetone. Sorporl-144 $(200 \mathrm{mg} / \mathrm{L})$, an emulsifier, was added to dilute the solution of each sample to $500 \mathrm{mg} / \mathrm{L}$. Then, $50 \mu \mathrm{g} / \mathrm{mL}$ of the test compounds were obtained by mixing $1 \mathrm{~mL}$ of drug solution with $9 \mathrm{~mL}$ of PSA culture medium. A bacterium tray of 5-mm diameter cut along the external edge of the mycelium was transferred to the flat surface containing the tested compound and put in equilateral triangular style in triplicate. Later, the culture plates were cultivated at $24.0 \pm 1.0^{\circ} \mathrm{C}$ and the extended diameters of the circles of mycelium were measured after $48 \mathrm{~h}$. Compared with the blank control, the relative inhibition percentage was calculated. All the experiments were performed in three replicates, and chlorothalonil (a commercial fungicide) was used as positive control. Activity grading indicators: Grade A: $\geq 90 \%$; Grade B: 70 90\%; Grade C: 50 70\%; Grade D: $<50 \%$.

\subsection{D-QSAR Analysis}

The 3D-QSAR model for finding out the correlation between the structure and activity of the target compounds was built using the CoMFA method of Sybyl-X 2.1.1 software. According to the literature [41], the antifungal activities of the title compounds against $P$. piricola were transferred to ED values through the formula:

$$
\mathrm{ED}=\lg \{\mathrm{I} /[(100-\mathrm{I}) \times \mathrm{MW}]\}
$$

where I was the inhibitory percentage at $50 \mu \mathrm{g} / \mathrm{mL}$ and $\mathrm{MW}$ was the molecular weight of the target compounds. Complete conformational optimization of each structure was performed using a conjugate gradient procedure based on the Tripos force field with termination convergence energy of $0.005 \mathrm{kcal} /\left(\mathrm{mol}^{*} \AA\right)$ and Gasteiger-Hückel charges. Compound $5 \mathrm{~b}\left(\mathrm{R}=o-\mathrm{CH}_{3} \mathrm{Ph}\right)$ was selected as the template molecule, in which the atoms 
marked with asterisks constituted the common superimposed skeleton (Figure 4), twentythree test compounds as training set were superimposed (Figure 5). CoMFA descriptors as independent variables and ED values as dependent variables. The cross-validation using the leave-one-out method was performed to obtain the cross-validated $q^{2}$ and the optimal number $N$ of components. Then, a non-cross-validation analysis under the optimal number of components was performed. The modeling capability was indicated by the correlation coefficient squared $r^{2}$, and the prediction capability was judged by the $r^{2}$ and $q^{2}$.

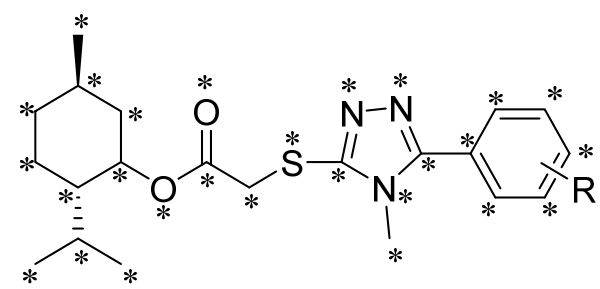

Figure 4. The asterisk skeleton of title compounds.

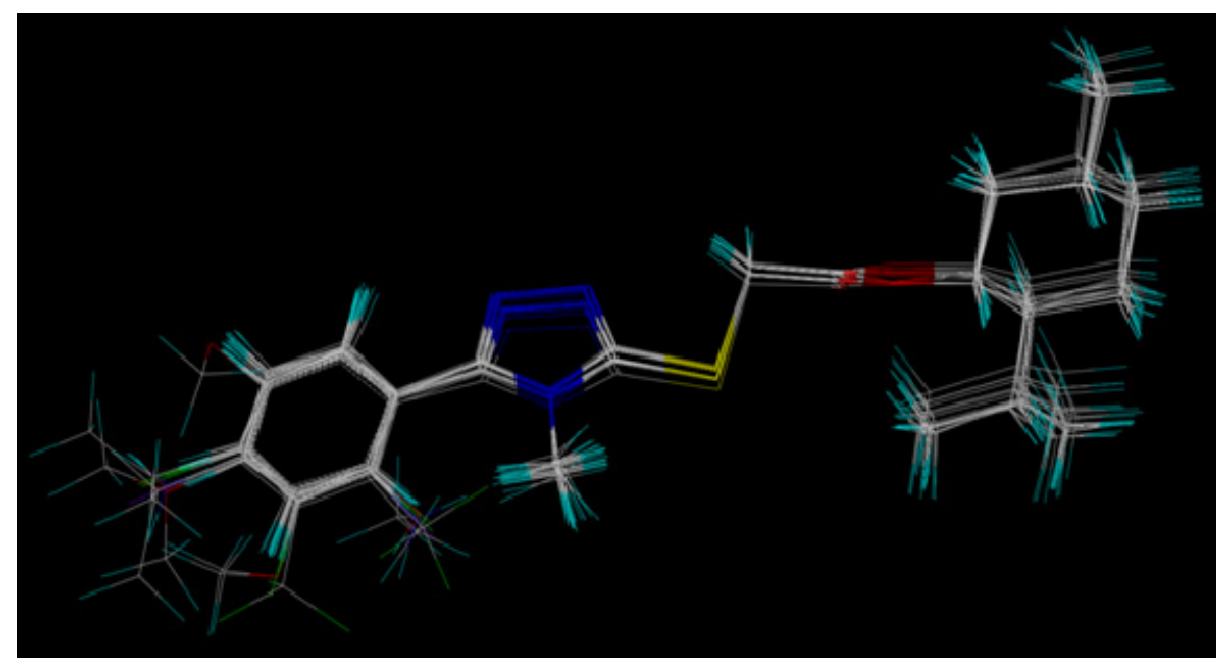

Figure 5. Superposition modes of compounds.

\subsection{Molecular Docking Analysis}

Molecular docking study was performed on Sybyl-X 2.1.1 program, using cytochrome P450 14 $\alpha$-sterol demethylase (CYP51, PDB ID 1EA1) as the receptor. The 3D structures of small-molecule ligands were obtained and minimized by the reported method [42]. Surflex-Dock was employed for the docking process, and the binding site of its original ligand was selected as the docking site. The docking result was also observed in the 2D diagrams drawn by LigPlot+ v2.1 (EMBL-European Bioinformatics Institute, Cambridge, United Kingdom).

\section{Conclusions}

In search of natural product-based antifungal agents, 26 novel menthol-based 1,2,4triazole-thioether compounds were synthesized. Their structures were confirmed by FT-IR, NMR, ESI-MS, and elemental analysis. The in vitro antifungal activity of the target compounds $\mathbf{5 a}-\mathbf{5 z}$ was preliminarily evaluated against eight plant pathogens at the concentration of $50 \mu \mathrm{g} / \mathrm{mL}$. The bioassay results indicated that some of the target compounds showed good inhibitory activity against the tested fungi, especially against $P$. piricola. Compounds $5 \mathbf{b}\left(\mathrm{R}=o-\mathrm{CH}_{3} \mathrm{Ph}\right), 5 \mathbf{i}(\mathrm{R}=o-\mathrm{Cl} \mathrm{Ph}), 5 \mathbf{v}\left(\mathrm{R}=m, p-\mathrm{OCH}_{3} \mathrm{Ph}\right)$ and $5 \mathbf{x}(\mathrm{R}=\alpha$-furyl $)$ had inhibition rates of $93.3 \%, 79.4 \%$, and $79.4 \%$, respectively, against $P$. piricola, much better than that of the positive control chlorothalonil. Compounds $5 \mathbf{v}\left(\mathrm{R}=m, p-\mathrm{OCH}_{3} \mathrm{Ph}\right)$ and $5 \mathrm{~g}(\mathrm{R}=o-\mathrm{Cl} \mathrm{Ph})$ held inhibition rates of $82.4 \%$ and $86.5 \%$ against C. arachidicola and $\mathrm{G}$. zeae, respectively, much better than that of the commercial fungicide chlorothalonil. Com- 
pound $5 \mathbf{b}\left(\mathrm{R}=o-\mathrm{CH}_{3} \mathrm{Ph}\right)$ displayed antifungal activity of $90.5 \%$ and $83.8 \%$, respectively, against $C$. orbicalare and F. oxysporum f. sp. Cucumerinu. Compound $5 \mathrm{~m}(\mathrm{R}=o-\mathrm{I} \mathrm{Ph})$ had inhibition rates of $88.6 \%, 80.0 \%$, and $88.0 \%$, respectively, against $F$. oxysporum f. sp. Cucumerinu, B. myadis and C. orbiculare. Furthermore, compound $\mathbf{5 b}\left(\mathrm{R}=o-\mathrm{CH}_{3} \mathrm{Ph}\right)$ displayed the best and broad-spectrum antifungal activity against all the tested fungi. To design more effective antifungal molecules against $P$. piricola, 3D-QSAR analysis was carried out using CoMFA method. A reasonable and effective 3D-QSAR model $\left(r^{2}=0.991, q^{2}=0.514\right)$ has been established. Molecular docking study revealed that there were $\mathrm{H}$ bonds and hydrophobic interactions between the target compounds and CYP51. Overall, compound $5 \mathbf{b}\left(\mathrm{R}=o-\mathrm{CH}_{3} \mathrm{Ph}\right)$ was a potential CYP51 inhibitor worthy of further study.

Supplementary Materials: The following are available online, Figures S1-S106: FT-IR, ${ }^{1} \mathrm{H}-\mathrm{NMR}$, ${ }^{13} \mathrm{C}-\mathrm{NMR}$, and ESI-MS spectra of compounds $\mathbf{2}$ and $\mathbf{5 a}-\mathbf{5 z}$.

Author Contributions: M.H. carried out the experimental work, participated in the discussion of biological activities, and wrote the paper; W.-G.D. and G.-S.L. designed the target compounds, directed and supervised the whole experimentation, discussed the biological activities, and revised the paper; B.-Y.L. participated in the discussion of biological activities, 3D-QSAR and molecular docking studies. All authors have read and agreed to the published version of the manuscript.

Funding: This work was financially supported by the National Natural Science Foundation of China (No. 31870556).

Institutional Review Board Statement: Not applicable.

Informed Consent Statement: Not applicable.

Data Availability Statement: Data are contained within the article or the Supplementary Data File.

Acknowledgments: The authors are grateful to the State Key Laboratory of Element-Organic Chemistry, Nankai University, China, for the bioassay test.

Conflicts of Interest: The authors declare no conflict of interest.

Sample Availability: Samples of the compounds are available from the authors.

\section{References}

1. Çam, M.; Yüksel, E.; Alaşalvar, H.; Başyiğit, B.; Şen, H.; Yllmaztekin, M.; Ahhmed, A.; Sağdıç, O. Simultaneous extraction of phenolics and essential oil from peppermint by pressurized hot water extraction. J. Food Sci. Technol. 2019, 56, 200-207. [CrossRef] [PubMed]

2. Skalicka-Woźniak, K.; Walasek, M. Preparative separation of menthol and pulegone from peppermint oil (Mentha piperita L.) by high-performance counter-current chromatography. Phytochem. Lett. 2014, 10, xciv-xcviii. [CrossRef]

3. Zaia, M.G.; Cagnazzo, T.O.; Feitosa, K.A.; Soares, E.G.; Faccioli, L.H.; Allegretti, S.M.; Afonso, A.; Anibal, F.F. Anti-Inflammatory properties of menthol and menthone in schistosoma mansoni infection. Front. Pharmacol. 2016, 7, 170. [CrossRef] [PubMed]

4. Desam, N.R.; Al-Rajab, A.J.; Sharma, M.; Mylabathula, M.M.; Gowkanapalli, R.R.; Albratty, M. Chemical constituents, in vitro antibacterial and antifungal activity of Mentha $\times$ Piperita L. (peppermint) essential oils. J. King Saud Univ. Sci. 2017, 31, 528-533. [CrossRef]

5. Elahe, J.; Seyed, E.H.; Abdorreza, M.N.; Leila, N. The effects of methylcellulose coating containing carvacrol or menthol on the physicochemical, mechanical, and antimicrobial activity of polyethylene films. Food Sci. Nutrition. 2021, 9, 2768-2778.

6. Dambolena, J.S.; López, A.G.; Cánepa, M.C.; Theumer, M.G.; Zygadlo, J.A.; Rubinstein, H.R. Inhibitory effect of cyclic terpenes (limonene, menthol, menthone and thymol) on Fusarium verticillioides MRC 826 growth and fumonisin B1 biosynthesis. Toxicon 2008, 51, 37-44. [CrossRef] [PubMed]

7. Murcia-Meseguer, A.; Alves, T.J.S.; Budia, F; Ortiz, A.; Medina, P. Insecticidal toxicity of thirteen commercial plant essential oils against Spodoptera exigua (Lepidoptera: Noctuidae). Phytoparasitica 2018, 46, 233-245. [CrossRef]

8. Zielińska-Błajet, M.; Pietrusiak, P.; Feder-Kubis, J. Selected monocyclic monoterpenes and their derivatives as effective anticancer therapeutic agents. Int. J. Mol. Sci. 2021, 22, 4763. [CrossRef]

9. Nagai, K.; Fukuno, S.; Omachi, A.; Omotani, S.; Hatsuda, Y.; Myotoku, M.; Konishi, H. Enhanced anti-cancer activity by menthol in HepG2 cells exposed to paclitaxel and vincristine: Possible involvement of CYP3A4 downregulation. Drug Metab. Pers. Ther. 2019, 34, 1-5. [CrossRef]

10. Ton, H.T.; Smart, A.E.; Aguilar, B.L.; Olson, T.T.; Kellar, K.J.; Ahern, G.P. Menthol Enhances the Desensitization of Human $\alpha 3 \beta 4$ Nicotinic Acetylcholine Receptors. Mol. Pharmacol. 2015, 88, 256-264. [CrossRef] 
11. Liu, B.; Fan, L.; Balakrishna, S.; Sui, A.; Morris, J.B.; Jordt, S.E. TRPM8 is the principal mediator of menthol-induced analgesia of acute and inflammatory pain. Pain 2013, 154, 2169-2177. [CrossRef]

12. Cantanhêde, S.M.; Amado, L.L.; da Costa, B.M.P.A.; Barbas, L.A.L.; Torres, M.F.; Hamoy, A.O.; da Paz, C.A.; da Silva Ferreira, C.B.; Lima, G.O.; de Sousa, J.R.; et al. Menthol exposure induces reversible cardiac depression and reduces lipid peroxidation in the heart tissue of tambaqui Colossoma macropomum. Aquaculture 2021, 541, 7368-7377. [CrossRef]

13. Otake, H.; Yamaguchi, M.; Ogata, F.; Deguchi, S.; Yamamoto, N.; Sasaki, H.; Kawasaki, N.; Nagai, N.; Nagai, N. Energy-dependent endocytosis is responsible for skin penetration of formulations based on a combination of indomethacin nanoparticles and L-menthol in rat and göttingen minipig. Int. J. Mol. Sci. 2021, 22, 5137. [CrossRef]

14. Sun, Y.J.; Wang, Z.W.; Wang, Y.X.; Tong, X.T.; Ke, Q.; Zhang, P. Synthesis and antimicrobial activity of 1,5-benzothiazepines incorporated with triazole moiety. Chin. J. Org. Chem. 2021, 41, 2361-2373. [CrossRef]

15. Amin, N.H.; El-Saadi, M.T.; Ibrahim, A.A.; Abdel-Rahman, H.M. Design, synthesis and mechanistic study of new 1,2,4-triazole derivatives as antimicrobial agents. Bioorg. Chem. 2021, 111, 104841-104853. [CrossRef]

16. Flavia, M.; Maurizio, B.; Federico, C.; Antonella, P. Chiral azole derivatives. Part 5: Synthesis of enantiomerically pure 1-[ $\alpha-$ (benzofuran-2-yl)arylmethyl]-1H-1,2,4-triazoles, antifungal and antiaromatase agents. Tetrahedron Asymmetry 2000, 11, 4895-4901.

17. He, Q.W.; Zhang, D.; Zhang, F.C.; Liu, X.H.; Feng, X.M. Asymmetric catalytic epoxidation of terminal enones for the synthesis of triazole antifungal agents. Organ. Lett. 2021, 23, 6961-6966. [CrossRef]

18. Bitla, S.; Gayatri, A.A.; Puchakayala, M.R.; Kumar Bhukya, V.; Vannada, J.; Dhanavath, R.; Kuthati, B.; Kothula, D.; Sagurthi, S.R.; Atcha, K.R. Design and synthesis, biological evaluation of bis-(1,2,3- and 1,2,4)-triazole derivatives as potential antimicrobial and antifungal agents. Bioorg. Med. Chem. Lett. 2021, 41, 128004-128012. [CrossRef] [PubMed]

19. Maddali, N.K.; Ivaturi, V.K.V.; Murthy Yellajyosula, L.N.; Malkhed, V.; Brahman, P.K.; Pindiprolu, S.K.S.S.; Kondaparthi, V.; Nethinti, S.R. New 1,2,4-triazole scaffolds as anticancer agents: Synthesis, biological evaluation and docking studies. ChemistrySelect 2021, 6, 6788-6796. [CrossRef]

20. Wu, C.J.; Wu, J.Q.; Hu, Y.; Pu, S.; Lin, Y.; Zeng, Z.; Hu, J.; Chen, W.H. Design, synthesis and biological evaluation of indolebased $[1,2,4]$ triazolo $[4,3-\alpha]$ pyridine derivatives as novel microtubule polymerization inhibitors. Eur. J. Med. Chem. 2021, 223, 113629-113644. [CrossRef] [PubMed]

21. Wang, C.S.; Huang, Q.; Wang, X.; Zhang, Y.T.; Ma, D.S.; Yu, Y.H.; Gao, J.S. Three new coordination polymers based on bis(4-(4H1,2,4-triazol-4-yl)phenyl)methane: Syntheses, structures, multiresponsive luminescent sensitive detection for antibiotics and pesticides, and antitumor activities. RSC Adv. 2019, 9, 42272-42283. [CrossRef]

22. You, Y.; Chen, Y.J.; You, C.H.; Wang, J.W.; Weng, Z.Q. Synthesis of 3-(tri/difluoromethyl)-1H-1,2,4- triazol-5(4H)-ones via the cyclization of hydrazinecarboxamides with tri/difluoroacetic anhydride. Org. Biomol. Chem. 2019, 17, 9343-9347. [CrossRef] [PubMed]

23. Mu, J.X.; Zhai, Z.W.; Tan, C.X.; Weng, J.Q.; Wu, H.K.; Duke, S.O.; Zhang, Y.G.; Liu, X.H. Synthesis and herbicidal activity of 1,2,4-triazole derivatives containing a pyrazole moiety. J. Heterocycl. Chem. 2019, 56, 968-971. [CrossRef]

24. Sun, G.X.; Yang, M.Y.; Zhao, W.; Sun, Z.H.; Wu, H.K. Crystal structure, DFT theoretical study and herbicidal activity of novel 1,2,4-triazole compound containing cyclopropyl group. J. Chem. Soc. Pak. 2015, 37, 567-573.

25. Li, S.M.; Tsai, S.E.; Chiang, C.Y.; Chung, C.Y.; Chuang, T.J.; Tseng, C.C.; Jiang, W.P.; Huang, G.J.; Lin, C.Y.; Yang, Y.C.; et al. New methyl 5-(halomethyl)-1-aryl-1H-1,2,4-triazole-3-carboxylates as selective COX-2 inhibitors and anti-inflammatory agents: Design, synthesis, biological evaluation, and docking study. Bioorg. Chem. 2020, 104, 104333-104346. [CrossRef] [PubMed]

26. Luo, D.X.; Guo, S.G.; He, F.; Wang, H.Y.; Xu, F.Z.; Dai, A.; Zhang, R.F.; Wu, J. Novel anthranilic amide derivatives bearing the chiral thioether and trifluoromethylpyridine: Synthesis and bioactivity. Bioorg. Med. Chem. Lett. 2020, 30, 126902-126922. [CrossRef]

27. Peng, F.; Liu, T.T.; Wang, Q.F.; Liu, F.; Cao, X.; Yang, J.S.; Liu, L.W.; Xie, C.W.; Xue, W. Antibacterial and antiviral activities of 1,3,4-oxadiazole thioether 4H-chromen-4-one derivatives. J. Agric. Food Chem. 2021, 37, 11085-11094. [CrossRef]

28. Nutt, M.J.; Yee, Y.S.; Buyan, A.; Andrewartha, N.; Corry, B.; Yeoh, G.C.T.; Stewart, S.G. In pursuit of a selective hepatocellular carcinoma therapeutic agent: Novel thalidomide derivatives with antiproliferative, antimigratory and STAT3 inhibitory properties. Eur. J. Med. Chem. 2021, 217, 113353-113372. [CrossRef] [PubMed]

29. Lin, G.S.; Bai, X.; Duan, W.G.; Cen, B.; Huang, M.; Lu, S.Z. High value-added application of sustainable natural forest product $\alpha$-pinene: Synthesis of myrtenal oxime esters as potential KARI inhibitors. ACS Sustain. Chem. Eng. 2019, 7, 7862-7868. [CrossRef]

30. Huang, M.; Huang, M.; Wang, X.; Duan, W.G.; Lin, G.S.; Lei, F.H. Synthesis, antifungal activity and 3D-QSAR study of novel acyl thiourea compounds containing gem-dimethylcyclopropane ring. Mol. Divers. 2021, 1-12. [CrossRef]

31. Wang, X.; Duan, W.G.; Lin, G.S.; Chen, M.; Lei, F.H. Synthesis, antifungal activity and 3D-QSAR study of novel nopol-based 1,3,4-thiadiazole-thioether compounds. Res. Chem. Intermed. 2021, 47, 4029-4049. [CrossRef]

32. Li, C.F.; Cen, B.; Duan, W.G.; Lin, G.S.; Wang, X.; Li, B.Y. Synthesis, herbicidal activity and three-dimensional quantitative structure-activity relationship (3D-QSAR) study of 4-methyl-1,2,4-triazole-thioether compounds containing natural styrene structure. Chin. J. Org. Chem. 2021, 41, 2485-2495. [CrossRef]

33. Yu, Y.P.; Duan, W.G.; Lin, G.S.; Kang, G.Q.; Wang, X.Y.; Lei, F.H. Synthesis, biological activity and three-dimensional quantitative structure-activity relationship (3D-QSAR) study of novel 4-methyl-1,2,4-triazole-thioethers containing gem-dimethylcyclopropane ring. Chin. J. Org. Chem. 2020, 40, 1647-1657. [CrossRef] 
34. Chen, M.; Duan, W.G.; Lin, G.S.; Fan, Z.T.; Wang, X. Synthesis, antifungal activity, and 3D-QSAR study of novel nopol-derived 1,3,4-thiadiazole-thiourea compounds. Molecules 2021, 26, 1708. [CrossRef]

35. Zhu, X.P.; Lin, G.S.; Duan, W.G.; Li, Q.M.; Li, F.Y.; Lu, S.Z. Synthesis and antiproliferative evaluation of novel longifolene-derived tetralone derivatives bearing 1,2,4-triazole moiety. Molecules 2020, 25, 986. [CrossRef] [PubMed]

36. Lin, G.S.; Duan, W.G.; Yang, L.X.; Huang, M.; Lei, F.H. Synthesis and antifungal activity of novel myrtenal-based 4-methyl-1,2,4triazole-thioethers. Molecules 2017, 22, 193. [CrossRef]

37. Lin, G.S.; Chen, Z.C.; Duan, W.G.; Wang, X.Y.; Lei, F.H. Synthesis and biological activity of novel myrtenal-derived 2-acyl-1,2,4triazole-3-thione compounds. Chin. J. Org. Chem. 2018, 38, 2085-2092. [CrossRef]

38. Tao, M.L.; Sun, H.J.; Liu, L.; Luo, X.; Lin, G.Y.; Li, R.B.; Zhao, Z.X.; Zhao, Z.X. Graphitized porous carbon for rapid screening of angiotensin converting enzyme inhibitory peptide GAMVVH from silkworm pupa protein and molecular insight into inhibition mechanism. J. Agric. Food Chem. 2017, 65, 8626-8633. [CrossRef]

39. Er, M.; Coskun, N. Substituent Effect on the Asymmetric Induction with (1R,2S,5R)-and (1S,2R,5S)-menthol Auxiliaries. J. Chem. Soc. Pak. 2010, 32, 198-208.

40. Saeed, A.; Ashraf, Z.; Nadeem, H.; Simpson, J.; Pérez, H.; Erben, M.F. An investigation of supramolecular synthons in 1,2,4triazole-3(4H)-thione compounds. X-ray crystal structures, energetic and hirshfeld surface analysis. J. Mol. Struct. 2019, 1195, 796-806. [CrossRef]

41. Liu, X.H.; Shi, Y.X.; Ma, Y.; Zhang, C.Y.; Dong, W.L.; Pan, L.; Wang, B.L.; Li, B.J.; Li, Z.M. Synthesis, antifungal activities and 3D-QSAR study of N-(5-substituted-1,3,4-thiadiazol-2-yl)cyclopropane- carboxamides. Eur. J. Med. Chem. 2009, 44, 2782-2786. [CrossRef] [PubMed]

42. Parkali, P.M.; Shyam Kumar, A.; Johanna, K.P.; Prodensia, T.S.; Turaga, S.; Shaiva, V.; Pujar, G.V.; Joshi, S.D.; Aminabhavi, T.M.; Dixit, S.R. Molecular docking and three-dimensional quantitative structure-activity relationships for antitubercular pyrimidine derivatives. Polycycl. Aromat. Compd. 2021, 1-14. [CrossRef] 